\title{
Modelling population dynamics based on experimental trials with genetically modified (RIDL) mosquitoes
}

\author{
Mario A. Natiello ${ }^{\dagger}$ and Hernán G. Solari ${ }^{\ddagger}$
}

13th February 2020

${ }^{\dagger}$ Centre for Mathematical Sciences, Lund University, Box 118, S 22100

LUND, Sweden. mario.natiello@math.lth.se (corresponding author)

${ }^{\ddagger}$ Departamento de Física, FCEN-UBA and IFIBA-CONICET, Argentina. solari@df.uba.ar

\begin{abstract}
Recently, the RIDL-SIT technology has been field-tested for control of Aedes aegypti. The technique consists of releasing genetically modified mosquitoes carrying a "lethal gene". In 2016 the World Health Organization (WHO) and the Pan-American Health Organization (PAHO) recommended to their constituent countries to test the new technologies proposed to control Aedes aegypti populations. However, issues concerning effectiveness and ecological impact have not been thoroughly studied so far. In order to study these issues, we develop an ecological model. It presents interdependent dynamics of mosquito populations and food in a homogeneous setting. Mosquito populations are described in a stochastic compartmental setup, in terms of reaction norms depending on the available food in the environment. The development of the model allows us to indicate some critical biological knowledge that is missing and could (should) be produced. Hybridisation levels, release numbers during and after intervention and population recovery time after the intervention as a function of intervention duration and target are calculated under different hypotheses with regard to the fitness of hybrids and compared with two field studies of actual interventions. This minimal model should serve as a basis for detailed models when the necessary information to construct them is produced. For the time being, the model shows that nature will not clean non-lethal introgressed genes.
\end{abstract}

Keywords: environmental impact, RIDL-SIT, compartmental model, epidemic risk, genetic modification, Aedes aegypti 


\section{Introduction}

The use of the Sterile Insect Technique (SIT) has been proposed to control populations of the mosquito Aedes aegypti (Ae ae.) because of public health reasons [1]. Ae ae. is the main vector of several viruses such as the Flavivirus producing Yellow Fever, Dengue and Zika, as well as the Alphavirus producing Chikungunya. The idea is not new and follows the success obtained with the fruit fly (Drosophila sp.) in agricultural settings.

The early attempts to use the method with $A e$ ae. sterilised by radiation ended in failure [2]. The evolution of SIT by the introduction of genetically modified insects, GMI, incorporating an autocidal gene [3] (termed RIDL-SIT, Release of Insects carrying a Dominant Lethal) was later considered to be a more promising technique for mosquito control than irradiated mosquitoes [4]. The RIDL-SIT technique for Ae ae. was developed recently and communicated in a series of papers [5, [6, 7, 8, 9, 10] (all references except the first one refer to actual field-tests).

The World Health Organization (WHO) recently promoted the realisation of evaluations of this technique [11] in the wake of the 2015 Zika pandemic in the Americas. More recently, the Food and Drug Administration (FDA-USA) authorised the realisation of such evaluations in the US [12]. In correspondence with technological and political developments, the Pan-American Health Organization (PAHO) endorsed WHO's recommendations for its member states. It is apparent that there exists a growing interest in performing tests of the RIDL-SIT technique in the field, despite the limited amount of available information and the uncertainty of impact assessments. Nevertheless, warnings were raised by scientists [13] that foresaw the ecological and social dimension of the problem, in correspondence with the considerations of social scientists regarding technological fixes [14].

In April 20-21, 2017 a scientific meeting was held at the Ministry of Public Health of Argentina with the support of PAHO, addressing the reasonability of

conducting such experiments in the country. The participants were the National health authorities, a PAHO representative, a group of scientists proposing integrated control technologies (including SIT), a group of scientists not involved in technological development and two representatives of the foremost RIDLSIT company. Despite the presence of a scientist representing the team developing the RIDL-SIT technique, some questions concerning impact assessment remained unanswered, being nevertheless relevant for the scientific community and the society as a whole, for example:

- Which lasting modifications of the environment will be produced by these tests?

- Will the population of vectors previous to the environmental intervention be re-established? If yes, how fast?

The goal of the present work is to address these and related issues focusing on the technique promoted by the WHO [11] concerning the transgenic mosquito 
OX513A [5, 6, 7, 8, 9, 10] (in the preliminary work [5] it is called LA513A) that was preliminarily evaluated in 12]. We will use the mathematical methods of stochastic population dynamics, which should in general be used before any intervention. We investigate the time-evolution of a mosquito population as a result of the proposed interventions, focusing on:

- The hybridisation of the released mosquitoes with the local populations as a result of genetic diffusion

- The expected ratio between released males and local males necessary to achieve different levels of control

- The recovery time of the new, hybrid, populations when the intervention ceases

- The reasons for the difficulties found in establishing such control systems

To achieve these goals we implement a stochastic model, able to deal with small population numbers, based upon the available information. Because of the scarcity of biological information concerning the released mosquitoes, we have kept the model as general as possible, avoiding the need to replace missing information with conjectures. We indicate the most noticeable missing information in our critical review of the data (see Section 2.3.2). It is also worth mentioning that construction of conclusions concerning some data available in the reports is occasionally inconsistent between different reports and/or existing biological knowledge.

This manuscript is structured as follows: In Section 2 we describe the biological background of the technique, including a critical review of the available data, as well as the mathematical methods supporting the simulation. Section 3 displays the outcome of the simulations and their relation to relevant biological questions. In Section 4 results of this work are compared with actual field observations, while Section [5] contains a general discussion and concluding remarks.

\section{Biological background and mathematical meth- ods}

\subsection{The RIDL-SIT technique}

The Sterile Insect Technique (SIT) was introduced in the past century for insect pest control. The goal is to release sterile males in sufficient number to reduce (control) the population by reducing the number of offspring. Sterility was originally achieved by radiation and it has been successful as part of the measures to control the Mediterranean fruit fly [15] (see below), the screwworm fly [16] and other insects regarded as pests (for a history see [17]). Hence, the idea of controlling populations of mosquito vectors of viral diseases by sterilisation emerged clearly. By 1962 Aedes aegypti was considered a mosquito that could 
be controlled with the technique. Field experiments were performed by Morlan et al. 2] but despite overwhelming releases of sterilised males in relation to the local mosquito population, no control was made evident. In consideration of this failure the technique had to be revised. Benedict and Robinson [4] discussed some conditions that a sterile insect technique should satisfy to be successful; we quote:

Apart from the obvious requisite of mating competitiveness, two criteria must be satisfied by any sterilisation method: (1) sterility must not be suppressed by complementation with any genotype present in field populations. Any variation in the expression of the sterility factor(s) following interaction with the large and heterogeneous genome of the field population will quickly lead to selection for non-sensitive females and fertile matings. (2) Absolute assurance of effectiveness must result from the sterilisation method in the factory setting.

These authors also recommended that

a first step for the future deployment of transgenic technology is to demonstrate the safety of release material in the absence of confounding concerns about the spread of alleles and drive elements. This is possible only if the released material is genetically sterile.

Several authors [18, 19, 20, 1] have indicated that density-dependent-effects can/will reduce the efficiency of SIT programs to the point of making it nonviable.

To overcome in part these population (ecological) effects, the RIDL-SIT technique was introduced. Transgenic mosquitoes were designed to have a late acting dominant lethal gene, LG, [5] following a technique developed for the fruit fly [3]. The original strains of modified mosquitoes were constructed using the Rockefeller strain of $A e$ ae., a strain that has been kept in captivity for many years and presents adaptation of its life-cycle to the laboratory [21, 22], as well as manifestly larger sizes and fertility than wild strains.

This work focuses on the technique developed upon the transgenic mosquito OX513A specified in the previous Section. The proposed strategy is to release solely male mosquitoes outnumbering the wild males and thus strongly influencing the fecundation of wild females. The offspring is expected to express the lethal gene and die before reaching fertile age. Thus, the introduced mosquitoes would, after one generation, disappear from the natural environment along with their offspring. The expected outcome in a closed environment (no adult immigration or emigration) is to control the original wild mosquito population.

The genetic engineering of the mosquitoes proceeds by inoculating eggs with molecular constructs to "induce tetracycline-repressible dominant lethality in both males and females" [5], producing four transgenic lines of modified mosquitoes which appear to be obtained by modifications at a single site in the genome. Three of these lines gave the desired mortality. One of these three 
presented increased mortality near pupation and was selected for mass production because of this desired attribute. According to [5], a penetrance of $93-97 \%$ was achieved, meaning that at the level of phenotype a $3-7 \%$ of the individuals carrying the gene do not manifest the early mortality. We found no studies concerning whether the individuals carrying the gene and arriving to adulthood transmit the trait of not-manifesting-the-modified-gene. For the case of the Mediterranean fruit fly, Gong et al. [3] followed one generation $(n=4590)$ concluding that survival to adulthood despite the lethal gene is not an inheritable trait. Such conclusion is inaccurate, a proper statement is that the probability of survival being inheritable lies in the interval $\left[0,5.9 \cdot 10^{-4}\right]$ with $95 \%$ confidence. The difference matters when this probability is multiplied by millions of released individuals. Nevertheless, the authors acknowledge that "the possibility of biochemical resistance to the lethal effector molecule remains a potential drawback to RIDL that is not a significant issue for radiation-based sterilization".

As part of the engineering process, a fluorescent marker was incorporated to the same modified gene. This marker facilitates identification of larvae of genetically modified mosquitoes.

The requisite of mating competitiveness [4] of the modified mosquitoes, is monitored by the quantity $C$, defined as the ratio between offspring (lower case) to adult males (upper case) of modified mosquitoes, $m / M$, normalised to the same ratio for the wild mosquitoes, $w / W$ :

$$
C=\left(\frac{m}{M}\right)\left(\frac{w}{W}\right)^{-1} .
$$

Low estimates values of $C$ will have to be compensated by performing larger releases. The estimation of $C$ performed in cage and field experiments produced values of 0.059 (Cayman Islands [7]) and 0.031 (Brazil, [9]). We leave aside a previous report for the Cayman Islands of $C=0.56[6]$ obtained in the context of trying to establish that mating of wild females with modified males was actually possible.

An additional source of uncertainty is the lifespan of modified adults in the wild. Mark, release and capture experiments were performed twice. For Ae $a e$. , the ability to produce reliable data in such experiments has been questioned. A large dispersion of results is obtained when trying to estimate dispersal distances, i.e., dispersal measurements appear to be greatly influenced by the measurement procedure [23, 24]. The reported results appear not to escape to this rule: survivals of 2 days are reported in [8] and of 5 days in [10]. In the first experiment the unmodified laboratory strain was released as well showing a 2.1 day survival in average with no significant differences with respect to the modified strain. The release of a control strain was not repeated in the second experiment, probably because of the urban setting in which it was performed. In contrast, the survival of modified mosquitoes tested against a strain collected in Chiapas (Mexico) termed Latin Wild Type, LWT, used as the basis of the FDA report [12, Appendix F, p. 6-7] contains only experiments in the laboratory, but none in the field. The experiment confirms the existence of significant differences in phenotype between LWT and the GMI in terms of fertility and 
hatch rate measured at the laboratory, the environment in which the GMI was developed. We have not found data testing survival rates of modified mosquitoes released in the field against the local wild population under similar conditions among the reported literature $([\underline{8}, 10,12])$.

\subsection{Mosquito, genetics and environment}

The populations of the tree hole mosquito Aedes aegypti are mainly limited by the development during the larval stage. The two main environmental factors that have been so far identified are temperature [25, 26] and food. It is expected that genetics will play a role but comparative studies of quantitative traits are not abundant. Studies comparing developmental rates of Ae ae. collected at different locations in Argentina and those of the Rockefeller strain (a laboratory strain), indicate [22] that there are significant differences, under the same temperature and laboratory feeding conditions, between local strains and the Rockefeller strain, as well as statistically significant differences between local (country wide) strains, despite being all of them identified in the same branch of the phylogenetic tree as hybrids between Aedes aegypti aegypti and Aedes aegypti formosus [27]. For example, the Rockefeller strain spends less time as larvae. Yet, the most substantial difference corresponds to the total number of eggs and the daily fertility which is five times larger for the Rockefeller strain than for the local strains. A recent study performed in Trinidad and Tobago [28] reports a fertility of local females raised in the laboratory, with local genetics, which is in line with the experiments reported in [22].

Any model (be it mathematically explicit or not) aimed at exploring the possible outcome of an environmental intervention, must include the reaction norms [29] of the local, the released and the resulting hybrid mosquitoes. The traits that have been identified so far are: hatching inhibition as a response to low levels of food (the Gillett effect) [30, 31, 32, 33, 34, 35, 36], duration of the larvae stage [37, 26, 38, 39, 40, 41], mortality of preimaginal forms [39, 40, 41] and fertility [42], directly associated to the body size of females. Furthermore, it has been observed that temperature and food are not independent factors [43].

Needless to say, the feeding behaviour of the larvae will alter the food resources in the container. Hence, food and phenotype must be considered as dynamical variables.

\subsection{Simple population dynamic model to evaluate effect- iveness and gene diffusion}

\subsubsection{Comments on previous models}

In Table 1 we display a few mathematical models that have bearing on the issues discussed in this work. The table classifies the models according to key features of our modelling strategy. Pop denotes the number of compartments. A large number of compartments allows for finer graining, since compartments differentiate groups of individuals with different characteristics, different dy- 
namical responses and/or different biology. Var indicates whether modelling is performed with continuous or discrete variables. Populations are counted with non-negative integers and are hence intrinsically discrete; continuous variables may constitute a fair approximation to large populations, but they are in conflict with the possibility of extinction of one or more compartments. Dyn indicates the type of dynamics. All models but the last in the table are deterministic (either differential equations or difference equations on discrete time). Such strategy forces in practice the use of continuous variables. SkeeterBuster, the last entry of the Table has an intermediate approach, being the time-lapse of the preimaginal stages stochastic. Eco describes whether the model takes into account the interaction of the populations with the environment. In fact, model parameters have a biological meaning, at least metaphorically, but in general they express the action of the environment on the populations, rather than a true interaction where the dynamics of the population modifies the environment as well. $S p$ indicates whether the spatial distribution is taken into account. Homogeneous environments can be modelled without spatial resolution, contrasting with inhomogeneous environments. Int: indicates whether introgression is considered. $G S$ : denotes the genetic strategy in the model ( $\mathrm{LG}=$ lethal gene, $\mathrm{Ge}=$ Generic) .

\begin{tabular}{|c|c|c|c|c|c|c|c|}
\hline Reference & Pop & Var & Dyn & Eco & Sp & Int & GS \\
\hline \hline$[44]$ & $5+1$ & $\mathrm{C}$ & Det & no & no & no & Ge \\
\hline$[5]$ & $1+$ delay & C & Det & no & no & no & LG \\
\hline$[45]$ & $1^{a}+$ delay & C & Det & no & yes & no & Ge \\
\hline$[46]$ & $2^{a}$ & C & Det & no & no & no & Ge \\
\hline$[47]$ & $2+$ delay & C & Det & no & no & no & Ge \\
\hline$[48, \underline{49}]$ & 2 & C & Det & no & yes & no & Ge \\
\hline$[50,51,52]$ & $b$ & D & Mix & yes & yes & no & Ge \\
\hline
\end{tabular}

Time is discrete.

${ }^{b}$ Not a compartmental model.

Table 1: Previous models. Pop: Number of populations, Var: Type of variables ( $\mathrm{D}=$ Discrete, $\mathrm{C}=$ Continuous), Dyn: Dynamics (Deterministic, Stochastic or Mix, see text), Eco: Ecology, $S p$ : indicates whether the spatial distribution is taken into account, Int: Introgression, $G S$ : Genetic strategy ( $\mathrm{LG}=$ lethal gene, $\mathrm{Ge}=$ Generic).

Concerning the central issues of this work, these models were not conceived to address the possibility of hybridisation and its eventual propagation in time, or the interruption of the interventions, and are hence insufficient to discuss the questions of interest for this study. The model in [45] includes a discussion about fitness of the RIDL released males but the context is how to compensate deficiencies in fitness with a higher release rate. The model proposed in [5] is the only one addressing the LG strategy. It has a low level of detail since it deals only with one population. Its goal was to address questions regarding the effectiveness of the proposed technique. FDA (Food and Drug Administration, US) 
[12] refers to this model in their assessment of the RIDL-SIT technique. Finally, most models treat the environment as "fixed". Even if biological parameters in the models are e.g., temperature dependent, the environment is imposed on the populations without feedback, being in this way outside the dynamical evolution. The possibility of (local) environmental modification originated in the time-evolution of the populations is absent.

A separate paragraph deserves the approach of SkeeterBuster (last entry of the table). The model produces detailed simulations based upon Focks' model CIMSiM [38, 53]. The original model was revised and improved to incorporate the discussion of some genetic aspects, mainly the fixation index, $F_{S T}$, in relation to spatial dispersion. The computation is performed on one neutral locus (two alleles). One locus accounts only for $(00,01,11)$ classes, meaning that wild and hybrids with no LG are considered identical. No hybridisation can be tracked in this form. The model is highly detailed (it tracks individual females) but its biological content targets other questions as compared with the present work. Additionally, the information required to run the model is difficult to obtain, excpet for the area of Iquitos (Perú), which is the main target for SkeeterBuster. Iquitos has been the locus of a long term research project in dengue and Aedes aegypti, but even in such conditions, parametrisation of the model is difficult: We read in the conclusions of [54], "CIMSiM and DENSiM are most sensitive to parameters for which little empirical information exists, and although this information can be extremely valuable for setting research priorities, it also places constraints on the use and interpretation of model outputs." When compared to early versions of aedesBA (a compartmental model that uses an intermediate level of information) no definite advantage was observed for any of the models [55]. The difference between modelling philosophies has been briefly discussed in $[56]$.

Mathematical models do not produce biological data, they articulate information to produce understanding and insight about what to expect given the present level of information. However, knowledge at times takes the form of knowledge of our ignorance: which information do we need that has not yet been produced? Measurements and experiments are made to match a preconceived form of articulation. Models call for new experiments to produce substantially superior forms of articulation than the irrationality in habits and intuition of (raw) empiricism.

The modelling situation presents the dilemma: should we refrain from modelling because of the lack of information to run our most detailed codes? Such a restriction has as consequence that models which are too limited to address the task (e.g., [5]), become the only decision-making contribution provided by the modelling community. The best is the enemy of the good, is a popular aphorism and Italian proverb (il meglio è l'inimico del bene) quoted by Voltaire. In what follows we present a viable option under the current circumstances. 


\subsubsection{Lack of information and modelling decisions.}

In the light of the limited or uncertain information concerning the RIDL-SIT technique, some modelling decisions were made, namely:

- The lack of information regarding the reaction norms to environmental conditions such as the availability of food have been decisive in the modelling decision of not considering high-level models such as [41] which incorporate phenotype variation in terms of environmental conditions. Highlevel models are sensitive to differences found in the phenotype for eggs collected at different places [56].

- The effect of the lethal gene seems to be apparent not before L4 stage and it is strongest at pupae level (see Table 1 in [5]). Hence, the relation between mortality to emergence related to unmodified pupae is adjusted so that for individuals carrying the lethal gene the probability of death as pupae is $\frac{19}{20}=0.95$ and the probability of adult emergence is $\frac{1}{20}=0.05$, since these are the only competing events in an exponential race [57] at pupa stage.

- Another substantial missing information concerns the contributions of dead larvae and pupae as a secondary source of food. Such contribution was incorporated in earlier models [38] based upon results in [58] and amount to a $12 \%$ recovery (the product of $40 \%$ nutritive value and $30 \%$ conversion of food in weight) according to these sources. Recycling of dead larvae was assumed to influence food availability during the developmental stages (see Section 2.3.6 for a description of the food cycle).

- Heritability of the survival trait despite the presence of the lethal gene has been set to zero.

- Mating effectiveness as compared with the wild strain has been rounded up to $C=0.06$.

- We have made no difference in the survival of adult males of different strains (other than the food deficit effect for individuals raised in the wild). None of the reported experiments checks lifespan of modified males vs. lifespan of wild males in the same wild environment under similar conditions. In our simulations, that target for a pre-established reduction level of the wild population, the mortality of released males will affect the release rate since a comparatively high proportion of males must be maintained throughout the process. Eventually, larger release rates may enter in conflict with the factory production capabilities. The assumption made here underestimates the strain put on the factory.

- Since there is no information regarding reaction norms of hybrids between local strains of $A e a e$. and the Rockefeller strain, we have initially assigned to all wild and hybrid mosquitoes the same reaction to food deficit stress using the set of reaction norms available for mosquitoes collected 
at Buenos Aires (Argentina) [41] corresponding, in the present model, to: pupation rate, fertility, mortality at the preimaginal stadiums, as well as the Gillett effect as quantitatively described in [35] and the mortality of adults. However, released adult mosquitoes (overwhelmingly males) are not raised under food deficit stress and their mortality rate is not corrected by food deficit but fixed as indicated in the previous point.

- In Subsection 3.2 we explore to some extent the dependence of hybridisation with respect to hybrid fitness in two ways: (a) We test two values for the mating efficiency of heterozygous males carrying one LG, O1, setting it equal to that of the wild strain or, alternatively, to that of homozygous males (carrying two copies of the LG). (b) For adult females, we tested doubling the mortality rate for the $O 1$ hybrids. In all cases, there is no point in considering hybrids not carrying a LG worse fitted than $O 1$ mosquitoes. For hybrids not carrying a LG (named Rock in Table 3) we considered a linear interpolation between the two extreme cases just described, modifying at the same time the mating efficiency of males and the mortality rate of females.

A model is not just a mere transcription of experimental findings but a logical construction as well. In our schematic construction we have considered the two food regimes described in [41] and the dependence of phenomenological traits with the logarithm of the food concentration. A region where the life form is impossible was considered for food concentrations below $2^{-8}$ of the optimal concentration values obtained in the laboratory (which correspond to 1 in these units). The region of high mortality was considered in the range $\left[2^{-8}, 2^{-6}\right)$ while the favourable region spans the interval $\left[2^{-6}, 1\right]$. In the most unfavourable region the only life form possible are dormant eggs inhibited from hatching by the Gillett effect (see Section 2.2), all other preimaginal forms have probability one of dying. In the transition region, the Gillett effect diminishes from total inhibition to no inhibition and the mortality moves from probability one to the values measured in laboratory experiments [41]. The fertility is considered to be proportional to the mass excess to the minimal form of the adult found at the $2^{-8}$ food concentration where it takes a zero value and grows to reach the measured value for $A e$ ae. in the region of optimal laboratory conditions [22]. Laboratory results indicate that the increase in size as measured by the wing length is linear with the logarithm of the food concentration [59] all along the considered region. Using the raw data of the experiment reported in [41, 59] we determined that the event rate as larvae (considering both death and pupation) increased linearly (with the logarithm of food concentration) in the favourable region and was approximately constant in the transition region.

\subsubsection{Stochastic Population Dynamics}

This approach to population studies assumes that the dynamics is adequately described by classifying the population into compartments $X_{j}, j=1, \cdots, N$ (described by nonnegative integer numbers), each compartment counting the 
number of individuals of a given type as a function of time. For example, mosquito subpopulations in the present study are represented by the class $D$ with elements $\{$ egg, larvae, pupae, adult female, adult male $\}$. Each element in $D$ will be further subdivided with respect to its genetic type according to $G$, with elements \{wild, hybrid, modified heterozygous, modified homozygous\}. Thus, the index $j$ can be thought of as listing the elements of the external product $D \times G$. Changes in time occur stochastically and are identified by events, labelled by the index $\alpha$, where $\alpha=1, \cdots, E$. The event $\alpha$ produces an instantaneous change in population compartment $j$ given by the integer $\delta_{j}^{\alpha}$. Note that events where an individual undergoes a transition from one compartment to another (e.g., pupation where a larva becomes pupa) have exactly two nonzero indices $\delta_{j}^{\alpha}$ : For $j=l$ (larva) $\delta_{l}^{\alpha}=-1$, while for $j=p$ (pupa) $\delta_{p}^{\alpha}=+1$. Also, death events have $\delta=-1$ for the corresponding compartment. Birth events (oviposition) may have $\delta>1$ (see Subsection 2.3.6). The time-evolution of the population, given an initial condition (here labelled as $t=0$ ) can be summarised as:

$$
X_{j}(t)=X_{j}(0)+\sum_{\alpha=1}^{E} \delta_{j}^{\alpha} n_{\alpha}(t)
$$

where $n_{\alpha}(t)$ is the number of events of type $\alpha$ that occurred up to time $t$ (a stochastic variable). This approach is called a Markov Jump Process (Markov since the only necessary information to compute the next change is the knowledge of the present state; Jump since the changes that take place are described by the integer $\delta_{j}^{\alpha}$ indicating a change in the number of individuals in compartment $j$ ). In the mathematical literature attention to these processes starts with Kolmogorov's foundational work [60] and its further elaboration by Feller 61]. A substantial effort to relate the stochastic description to deterministic equations was performed by Kurtz [62, 63, 64].

Traditionally, attention was focused on the dynamics in population space, rather than event space. In the case of probability rates that are linear in the subpopulations both approaches are quite interchangeable 65]. However, event space is more appealing for a general understanding. For example, when the dynamics is affected by introducing a treatment or strategy regarded as an event (think e.g., of vaccination in an epidemic disease) it will be important for policy reasons (logistics, costs, etc.) to count the number of occurrences of that event, rather than attempting to sort it out by reversing the information contained in the population set $X=\left\{X_{j}(t), j=1, \cdots, N\right\}$.

The Markov assumption leads to defining probabilities per unit time $W_{\alpha}$ (in the sequel called rates or probability rates) for each event. In this work it will suffice to consider that these probability rates depend only on the population $X$ at each given time (other dependencies will be handled along the way). In a small time-interval $h$ the probability of occurrence/non occurrence of event $\alpha$ is the pair $\left(W_{\alpha}(X) h+o(h), 1-W_{\alpha}(X) h+o(h)\right)$, where $o(h)$ is a quantity that goes to zero with $h$ faster than linearly.

The process is further modelled implementing the Feller-Kendall algorithm [66, 67] that can be summarised in the following steps [65]: 
i. Starting at $t=0$ or immediately after an event has occurred, the waiting time to the next event is exponentially distributed, with rate $R=$ $\sum_{\alpha} W_{\alpha}(X)$ equal to the sum of all event probability rates, where $X$ refers to the population values after the most recent event has occurred (or to $X(0)$ ). The time $\tau$ to the next event is simulated by picking an exponential random deviate with rate $R$.

ii. Given that an event has occurred at time $\tau$, the probability of it being event $\alpha$ is

$$
P(\alpha / \text { event at } \tau)=\frac{W_{\alpha}(X)}{\sum_{\alpha} W_{\alpha}(X)}=\frac{W_{\alpha}(X)}{R} .
$$

Choosing an arbitrary order for the events, the event that took place at $\tau$ is simulated by picking a uniformly distributed random number $Y$ in $[0, R]$ and checking to which event in the order it corresponds. For example, if $\sum_{\alpha=1}^{K} W_{\alpha}(X)<Y<\sum_{\alpha=1}^{K+1} W_{\alpha}(X)$, we assign the occurrence to event $K+1$.

iii. Populations are updated according to $\delta_{j}^{\alpha}$ for the assigned event and a new cycle 1-3 is computed until the final simulation time is reached.

\subsubsection{Schematic description of the model}

The setup in consideration is that within a limited natural population of $\mathrm{Ae}$ ae., male adult mosquitoes are introduced (with an error of 1 female every 4300 releases [9]). These mosquitoes differ in two ways from the wild population. Firstly, they have two copies of a dominant LG (not present in the wild population) that induces massive death of the offspring before adult stage. The released mosquitoes have been previously raised in the laboratory, from the Rockefeller strain of Ae ae., a laboratory-adapted strain. These mosquitoes are genetically different from the original wild population. If the totality of the offspring does not die before reproduction, there will be some degree of population mixing and genetically modified individuals (relative to the original wild population) could arise.

Hence, to describe this problem a model has to be designed covering three goals:

i. Describe the wild population with sufficient accuracy taking care of environmental constraints.

ii. Take care of the evolution of the LG in the offspring along generations. A released homozygous mosquito mating with a wild female (where the corresponding gene is not lethal) will yield heterozygous offspring and any eventual subsequent offspring will propagate this special gene according to the mendelian rules.

iii. Take care of the mixing between the natural population and the released, genetically different, mosquitoes (mixing of other genes apart from the lethal one). 
Within this approach, the specification of the model requires (a) to identify the populations (compartments) participating in the dynamics and (b) to specify the events that define the dynamics, their reach, biological content and environmental conditions.

As mentioned in the previous Subsection, the subpopulations involved are chosen according to the developmental stages (egg, larva, pupa, male adult and female adult). In the absence of released mosquitoes the events governing the population dynamics are egg hatching and mortality, larval pupation and mortality, pupa emergence and mortality, fecundation (mating) and oviposition and finally adult mortality. These events take care of modelling goal i.

All compartments are further classified according to their genetical content. There is a compartment for the wild lineage and three for the mixed lineage where 0,1 or 2 copies of the LG may be present. This population space allows for dealing with modelling goal ii.

Finally, each subpopulation has attached a number in $[0,1]$ representing the average percentage of foreign genetic material. Released mosquitoes contribute with 1 , wild mosquitoes with 0 and the mixing propagates according to the law of independent assortment of alleles [29]. This takes care of modelling goal iii.

A detailed scheme of compartments and relating events is shown in Figure [1.

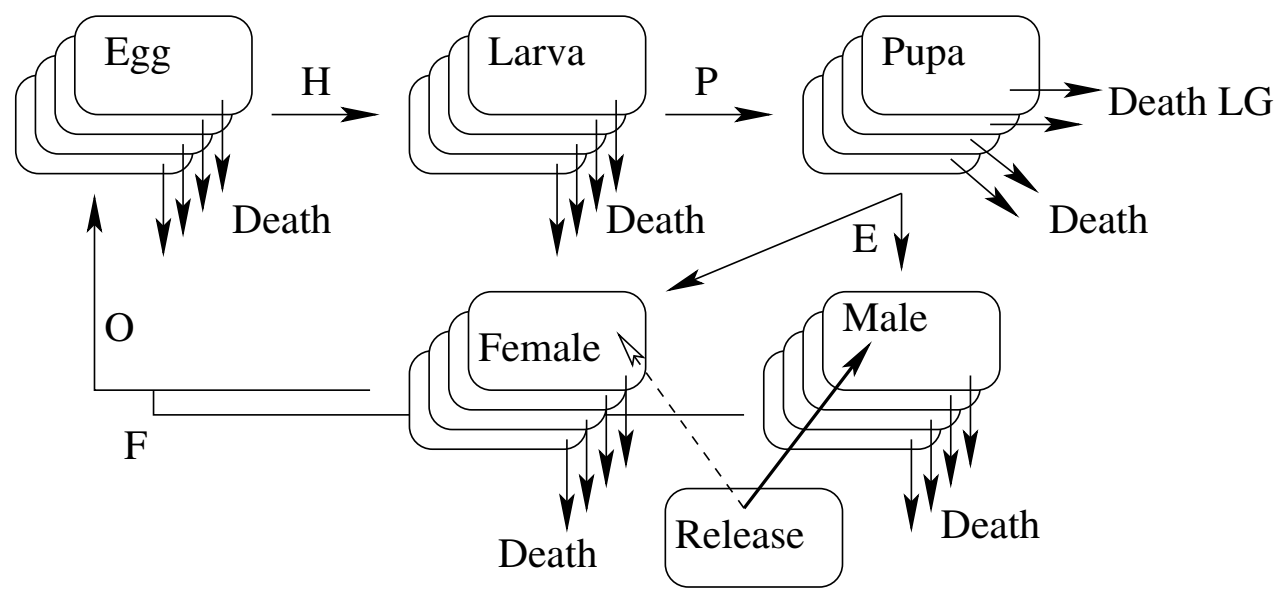

Figure 1: Compartments and events. The four copies of each compartmental stage correspond to the cases wild, 0,1 , or 2 copies of the LG. H: hatching, P: pupation, E: emergence, F: fecundation (mating), O: oviposition, Death: Death rate (specific for each stage), Death LG: modified death rate at the pupa stage due to the presence of the LG. 


\subsubsection{Food dynamics}

Most rates, especially larval pupation and mortality rates, depend on the availability of food. Following the model in [Romeo Aznar et al. 56] we assume that the totality of oviposition sites can optimally host $l_{o p t}$ larvae (grown to their potential size), that may feed unrestrictedly. At the same time, food in the sites is produced by (environmental) bacterial activity, it degrades at a certain rate $u$ (also depending on the environment, in this work $u=\frac{1}{\text { days }}$ ) and it is consumed at a pace depending on the amount of larvae. Dead larvae and pupae are to some extent recycled as available food, as mentioned in Section 2.3.2. Let $\frac{P_{f}}{C_{f}}$ denote the ratio of produced to consumed food. Initially, we set its value to the deterministic equilibrium value, an environment-dependent estimate. We denote the leftover food (relative to consumed food) as $L_{f}$. In conditions of food scarcity, $L_{f}=0$. The dead larvae or pupae recycled as food is denoted as $X_{l}$. This last parameter is initially zero and it is updated at every larval or pupal mortality event (see below). The ratio $\frac{P_{f}}{C_{f}}$ indicates the environmental scarcity conditions: if lesser than one, there is no food available for the larvae to reach the potential weight at pupation (this ratio enters in several steps of the modelling procedure). The food-cycle for each simulation time-step $\tau$, where the initial total larva population is $L$ and the ideal estimated capacity of the oviposition sites is $l_{\text {opt }}$, proceeds as follows:

$$
\begin{aligned}
L_{f} & =\max \left(\frac{P_{f}}{C_{f}}-1,0\right) \\
\frac{P_{f}}{C_{f}} & =L_{f} \exp (-u \tau)+\frac{l_{\text {opt }}+X_{l}}{L} \\
X_{l} & =X_{l} \exp (-u \tau)
\end{aligned}
$$

At each time-step, the values of $L$ and $X_{l}$ are updated and all above quantities recalculated. The influence of the food in mosquito dynamics is described by larval event rate $L E(x)$, mortality probability as larvae and pupae $M L(x)$, hatching inhibition $G L(x)$ and fertility rate $F T(x)$

$$
\begin{aligned}
L E(x) & =\max (0.025,0.25269+0.031974 x) \\
M L(x) & = \begin{cases}1 & x<-8 \\
\max (0.033,0.033-0.4835(x+6 .)) & x \geq-8\end{cases} \\
G L(x) & = \begin{cases}1 & x \geq-6 \\
(1 .+0.5(x+6 .)) & -8 \leq x<-6 \\
0 & x<-8\end{cases} \\
F T(x) & =\max (0 ., 0.127742(x+8 .))
\end{aligned}
$$

where $x=\log _{2}\left(\frac{C f}{P f}\right)$. The fertility rate changes the fertility of the emerging females, and then the average fertility of females (see below). The values for the reaction norms correspond to the values reported in [41]. In addition, the average lifetime as adult is corrected as $m_{a}=\frac{m_{a 0}}{1-M L(x)}$ obtaining in this form 
a reasonable correspondence between times measured at the laboratory (see for example [28]) and in field experiments (for example [37]). Released male mosquitoes, that had no food deficit while raised, were set to have average lifetime $m_{a 0}$ (see values below).

\subsubsection{Specific details}

The theoretical basis of the model when it comes to the population dynamics of Ae ae. refers to [56, 68, 23]. The problem is quasi-linear [65] in the populations, meaning that (a) all events that decrease a subpopulation produce a decrease in one unit in only one population compartment and (b) all non constant rates can be written in the form $W_{\alpha}=m_{\alpha}^{i} X_{i}$, for the relevant population $X_{i}$. However, the coefficients $m_{\alpha}^{i}$ need not be all constant.

The events hatching and pupation are assumed to have the same coefficient for all four compartments of the participating subpopulation. All these events pick one individual from the original subpopulation and passes it to the corresponding subpopulation of the next stage. Pupa emergence is less likely for the compartments carrying 1 or 2 copies of the LG (see below for the explicit values). Emergence events are randomly assigned to yield $50 \%$ males and females. Each mortality event simply decreases the corresponding subpopulation in one unit. Except in the case of pupa mortality, where the effect of the LG also distinguishes two cases, the mortality coefficient is taken to be the same along each stage. The pupa mortality is much higher for the compartments having 1 or 2 copies of the LG (see Appendix I for details of the estimates). As indicated in Section 2.3.2, adult mortality was assumed to be equal for all strains. This modelling decision may overestimate the size of non wild subpopulations, an effect that may be mitigated by larger releases.

The release was acted weekly during the treatment period, following the implementations of this technique discussed above, that only report weekly releases. The release goals of [7] were a relation of $10: 1$ in adult males (later increased to $25: 1$ ) and $50 \%$ fluorescence in the larvae from ovitrap-collected eggs. The initial size of the release is therefore set to be 11 times larger than the adult male population in our computations. Later release events are increased or reduced adaptively every week, in order to adjust the weekly proportion of eggs with LG against a target value. There is however a maximum release size corresponding to the maximal capacity of the production plant. In this work it is set to 100000 individuals per release. Release is intended to concern only modified males, but a few females are released as well, since the accuracy of the separation technique is limited (one female every 4300 releases [9]).

The model simulates a closed environment, i.e., no emigration/immigration of adult individuals to and from adjacent areas is taken into account. Clearly, such effects could only delay or reduce any effects of the release since immigrants, at least initially, would be wild individuals while emigrants could be any among released, wild and mixed individuals.

In this implementation, fecundation (mating) is not treated as a separate event. Instead, we assume that given that the adult male population is nonzero, 
all females are fecundated immediately after emergence. Thus, each emerged female has two associated indices, corresponding to the adult female compartment (inherited from the pupa) and the fecundating male compartment. The latter is chosen randomly among the four male subpopulations, weighing the size of the subpopulation with two copies of the LG with a factor 0.06 as described in the previous Sections. We assume hence that this reduced effectiveness is coupled to a high proportion of non-wild genes and to the homozygous property.

The index pair given by fecundation is used to propagate to the offspring both the LG and the percentage of introduced genes from the released population (according to the independent assortment of alleles [29], i.e., with the average of parental percentages). The propagation takes place in the oviposition event that adds individuals to the egg population (distributed among the four egg compartments according to mendelian rules) without decreasing any other subpopulation. The amount of eggs laid by each female at each oviposition depends on temperature and on the size of the female, which in turn depends on the environmental food availability. In average, wild mosquitoes collected in Argentina and reared in an environment with optimal food availability $\left(\frac{P_{f}}{C_{f}}=1\right)$ at $T=26 C$, lay 2 eggs per day [22]. Hence, the number of eggs per oviposition is $\delta_{e g g}=\frac{2}{m_{\text {ovi }}(26) F T(1)} F T\left(\frac{P_{f}}{C_{f}}\right) \equiv c_{l a y} F T\left(\frac{P_{f}}{C_{f}}\right)$. Once a female has emerged, its size is fixed as well as its egg-laying capacity, both by the value of $\frac{P_{f}}{C_{f}}$ at emergence (which varies among events according to food dynamics). Thus, at each emergence event, $\delta_{e g g}$ is updated as follows:

$$
\delta_{e g g}:=\frac{\delta_{e g g} F+c_{l a y} F T\left(\frac{P_{f}}{C_{f}}\right)}{F+1},
$$

where $F$ is the total number of adult females existing previous to the actual emergence. This is a sort of "moving average".

Dead larvae and pupae are partially recycled by the environment within the hatching sites. For each death event we add to $X_{l}$ the amount $\Delta l=x l \frac{F T\left(\frac{P_{f}}{C_{f}}\right)}{F T(1)}$. The quotient estimates the reduced size of each dead larva or pupae relative to ideal food conditions. The values $x l=0,0.12$ (as suggested by results in [38] and [58], see Section 2.3.2] and 0.5 were tested.

Most rates are known to be temperature dependent [26, 68, 43]. In this work we fixed temperature at the value $T=26 C$, corresponding to a yearround stable tropical environment. The coefficients for the different rates (in units of $(\text { days })^{-1}$ ) are displayed in Table 2, along with the references where the model structure and rates have been discussed:

\subsubsection{Hybridisation degree}

The inheritance of genotype is acted at fecundation. On one hand, the different subpopulations are classified in four compartments, depending on they being "purely wild" or mixed (e.g., a wild female mating a released male), with 0, 1 or 2 copies of the LG (here labelled $W, M_{0}, M_{1}, M_{2}$ ). When the treatment 


\begin{tabular}{|c|c|}
\hline Event & Rate \\
\hline \hline Hatching $^{a, b}$ & $m_{e \rightarrow l}=\left(-0.0167105+0.03866 \exp \left(\frac{T+7.26}{16.47906}\right)\right) G L\left(\frac{P_{f}}{C_{f}}\right)$ \\
\hline Egg mortality $^{a, c}$ & $m_{e}=0.01$ \\
\hline Pupation $^{b}$ & $m_{l \rightarrow p}=L E\left(\frac{P_{f}}{C_{f}}\right)\left(1-M L\left(\frac{P_{f}}{C_{f}}\right)\right)$ \\
\hline Larval mortality $^{b}$ & $m_{l}=L E\left(\frac{P_{f}}{C_{f}}\right) M L\left(\frac{P_{f}}{C_{f}}\right)$ \\
\hline Pupa emergence no LG $^{b}$ & $m_{p \rightarrow a}=0.5787\left(1-M L\left(\frac{P_{f}}{C_{f}}\right)\right)$ \\
\hline Pupa mortality no LG $^{b}$ & $m_{p}=0.5787 M L\left(\frac{P_{f}}{C_{f}}\right)$ \\
\hline Pupa emergence with LG & $m_{p l \rightarrow a}=\frac{1}{20}\left(m_{p}+m_{p \rightarrow a}\right)=\frac{1}{20} 0.5787$ \\
\hline Pupa mortality with LG $_{p l}=\frac{19}{20}\left(m_{p}+m_{p \rightarrow a}\right)=\frac{19}{20} 0.5787$ \\
\hline Adult mortality $^{b}=\frac{0.04}{\left(1-M L\left(\frac{P_{f}}{C_{f}}\right)\right)}$ \\
\hline Adult mortality $(2 \mathrm{LG})^{d}$ & $m_{a}$ \\
\hline Oviposition $^{b}$ & $m_{a v i}=0.03154 \exp \left(\frac{T-4.7511}{10.580}\right)$ \\
\hline
\end{tabular}

${ }^{a}$ See [68]. ${ }^{b}$ See [56, 59]. ${ }^{c}$ See [69]. ${ }^{d}$ See [12].

Table 2: Events and event rates. (LG stands for Lethal Gene)

starts, the first generation offspring of the released males with wild females will invariably land on compartment $M_{1}$ but later generations may mix further, since pre-adult mortality of the mixed offspring is not total. In any case, the offspring falls always in one of the four compartments.

Regarding the rest of the genetic material, the average degree of mixing $R$ of each compartment is computed in several steps. This value changes for each individual at fecundation/oviposition. Further, when new individuals enter a compartment, the compartmental average for $R$ changes accordingly and this modification propagates along the life cycle. Wild population has initial mixing value 0 , while released mosquitoes have value 1 , corresponding to $100 \%$ Rockefeller-strain genetic material. Following the law of independent assortment of alleles, offspring has a mixing equal to the average of the parental values, $R_{o}=\frac{1}{2}\left(R_{m}+R_{f}\right)$. Laid eggs (eq. 1), however, distribute among compartments according to mendelian laws. Finally, the average mixing of the affected compartments is consequently moved as,

$$
R_{n}:=\frac{R_{n} X(n)+R_{o} E}{X(n)+E},
$$

where $E$ is the amount of eggs (with inherited mixing $R_{0}$ ) belonging to compartment $n$ and $X_{n}$ is the preexistent number of eggs in that compartment (with previous average mixing $R_{n}$ ). 


\section{$3 \quad$ Results}

The code used for the simulations is deposited on GitHub. See https:// github.com/MNatiello/gene-diffusion-mosquito

Model simulations were performed using the following scheme:

- The initial wild populations and environmental food conditions were set to the deterministic equilibrium value (see Appendix II for a description).

- For a transient time $t_{T}=156$ weeks (about 3 years), the wild population was allowed to proceed without interference. The population data was saved to start the next simulation.

- After the previous transient, intervention was started, for a duration $t_{I}=$ 52 or 260 weeks for the results of Subsection 3.1 and $t_{I}=116$ weeks for those in Subsection 3.2 .

- After finishing the intervention, the system was simulated for another 156 weeks.

- The exhibited results are the average of 10 runs of the previous scheme.

- The short treatment was targeted to a weekly fluorescence ratio of 0.5 and the longer ones to ratios of 0.5 and 0.65 between eggs hatching to fluorescent vs total number of hatched eggs (per week).

- The recycle coefficient $x l$ at equilibrium was set to 0.12 for the short treatment while for the long treatment we illustrate the dynamics with $x l=0,0.12$ and 0.5 .

- There is no allowance for differentiated hybrid adult fitness (other than in Subsection 3.2).

\subsection{Summary of results}

We illustrate the simulations results with a few graphs indicating the timeevolution of the displayed properties. The abscissa of all graphs has units of days, counted from the beginning of the simulation. The transient, treatment and final portions are evident.

In Figure 2 we display the time-evolution of the number of adult females carrying no LG for the short treatment (1 year), where the ratio of eggs with LG to total number of eggs each week is targeted to $50 \%$. The recycling coefficient of dead larvae and pupae is set to 0.12. The drop in population size during the intervention is evident. Initially, the drop is more intense since the adjusting mechanism to target fluorescent eggs is slow (see below for a discussion). For

comparison, we plot one single simulation run superimposed to the average of 10 simulation runs.

Figure 3 displays the time-evolution of the number of adult females carrying no LG for the long treatment (5 years), where the ratio of eggs with LG to total 


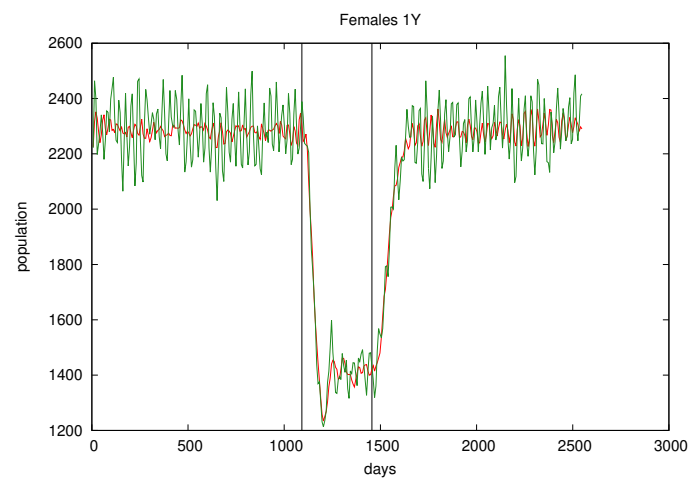

Figure 2: Females with no LG, 1-year treatment, $50 \%$ eggs with LG. Vertical lines indicate beginning and end of treatment. Green: single simulation line, Red: average of 10 simulation runs.

number of eggs each week is targeted to $50 \%$ or $65 \%$. Same considerations as in the previous picture hold. The three curves correspond to different values of the recycling coefficient of dead larvae and pupae (see caption).
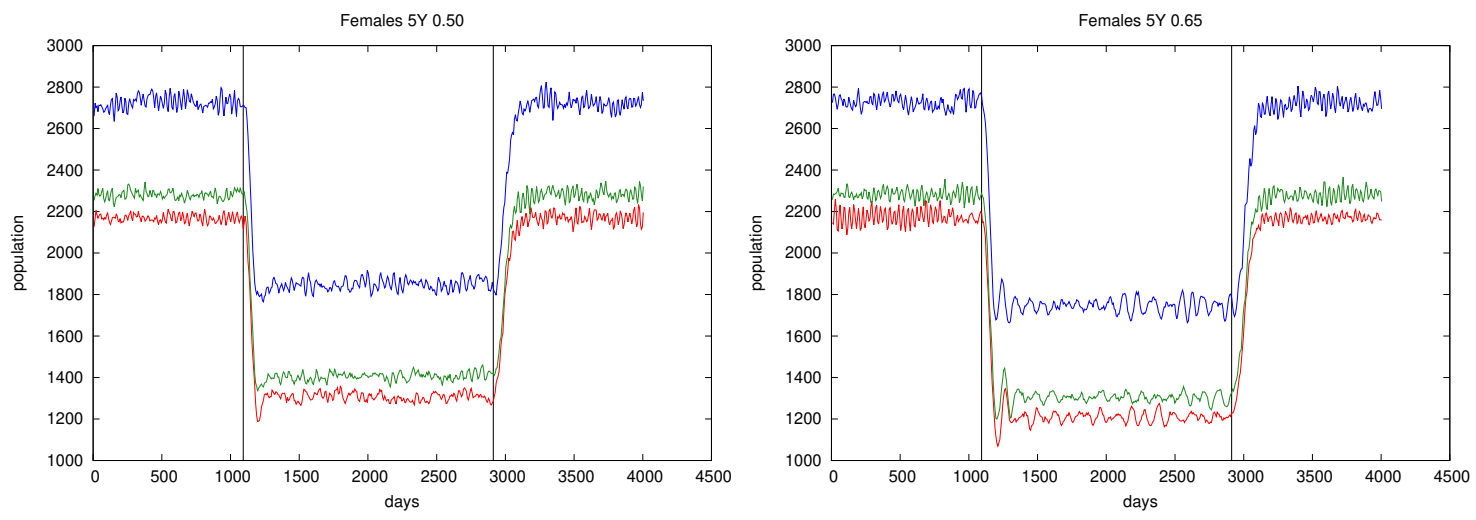

Figure 3: Females with no LG, 5-year treatment, $50 \%$ (left) or $65 \%$ (right) eggs with LG. Curves correspond to values 0 (red), 0.12 (green) or 0.5 (blue) for the recycling coefficient of dead larvae and pupae. Vertical lines indicate beginning and end of treatment.

The target goal we attempt to simulate follows [7] and aims to obtain a 50 or $65 \%$ proportion of laid eggs every week carrying one or two copies of the LG. The idea of monitoring eggs in order to regulate the treatment may be simple, but the control procedure is quite involved. On one hand, eggs have to be hatched in order to observe the larval fluorescence, meaning that the information about the system in actual implementations is obtained with certain delay (of the order 
of days). On the other hand, the control is acted by modifying the release, i.e., mainly adult males. In the model, it takes two (target 50\%) or three (target $65 \%$ ) weeks before the release of adults propagates to a modified proportion of eggs of about half the desired target.

The simulations display a reduction of adult females (and all other subpopulations except adult males) during the treatment period. This reduction could be said to be environment dependent, since it varies with the ability of the oviposition/breeding sites to recycle dead larvae and pupae into more food. There is an initial drop passing the target since the control procedure is indirect (eggs are monitored but the only modification to the system acts through the introduction of adult males). The other target in [7] is satisfied in excess, as seen in Figure 4, left. The adult male ratio is permanently above $10: 1$ during the treatment, while in the most demanding treatments it can be as high as $40: 1$.

After finishing the treatment, the system returns to a situation that is comparable with the initial equilibrium condition, the main difference being displayed in Figure 4, right. This figure displays the time-evolution of the proportion of alien genes in the population, a measure for genetic diffusion. Only the recycling coefficient 0.12 is plotted, since the differences with the other situations are minor. Three of the four female compartments die out, remaining only the hybrid compartment with no LG. The new equilibrium condition, although being quantitatively similar to the one before treatment, bears now different mosquitoes, the difference being larger for the longer and more demanding treatments.
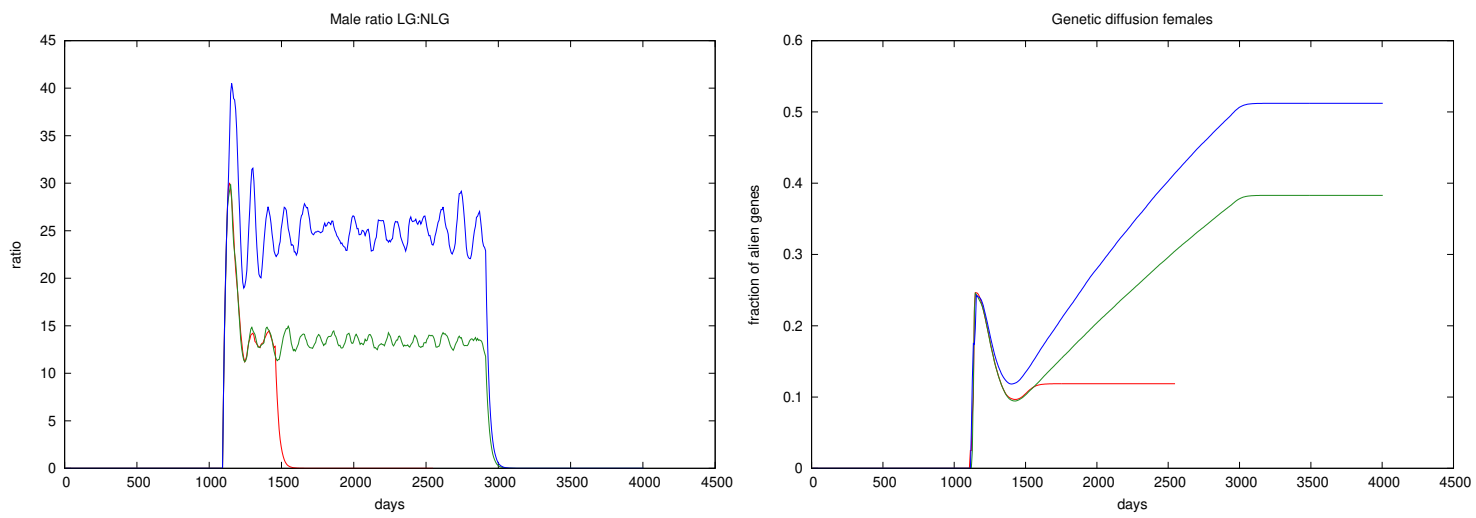

Figure 4: Adult male ratio with vs without LG (left) and genetic diffusion, i.e., the proportion of alien genes in the female population (right). Red: 1 year, target 0.5. Green: 5 years, target 0.5.Blue: 5 years, target 0.65 .

The fact that the system returns to its previous equilibrium value can be understood in different levels. From the simulation viewpoint, the system was assumed to be in a stable equilibrium before treatment. Despite the more or less drastic influence of the treatment, it eventually returns to this equilibrium 
when left alone. However, simulations with large weekly releases (about eight times larger than the maintenance release for the 0.5 target) under a sufficiently long time (more than one year) shift the final equilibrium to a smaller permanent population with $100 \%$ Rockefeller hybridisation homozygous in the LG. In Appendix D we comment on the effect of constant weekly release of different sizes.

From the modelling viewpoint, we pointed out before that there exists no knowledge about the adaptability of the modified hybrid mosquito populations to the actual environment. This possibility is consequently not considered in the model. Similarly, it is not known whether mosquitoes with $50 \%$ of the genetic content of the Rockefeller strain differ from the wild strain in their behaviour and their efficiency as vectors of viral diseases.

\subsection{Dependence of the degree of hybridisation with hybrid fitness.}

The target value was adjusted to obtain a control (reduction) of the viable-egg population close to $70 \%$, resulting in a value of 0.65 for our targeting strategy. In Table 3 we show the achieved population control, the hybridisation level, $R$, and its range (given as the extreme values of $R$ over 10 runs of the simulation) for different control targets and recycling level of food, while using different hypotheses regarding the fitness of the hybrid adult population. $O 1$ are hybrids with one LG and Rock is the hybrid with no LG. At the end of the runs all the population was of Rock type. The intervention were chosen to be 116 weeks long in accordance with the intervention at Juazeiro [70].

\subsection{Report of simulated scenarios: Questions and answers.}

We summarise our findings with a set of questions and answers. The first two questions are those present in the Introduction and originally triggering this study.

A. Which lasting modifications of the environment will be produced by these tests?

The main modification is the hybridisation of the released mosquitoes with the local populations as a result of genetic diffusion. The degree of hybridisation depends both on the duration of the treatment and its intensity (target), as well as on the (environmental) differences in food dynamics.

B. Will the population of vectors previous to the environmental intervention be re-established?

The recovery time of populations can be counted in months. There is no difference in population sizes before and after the treatment for the targeted programmes. 


\begin{tabular}{|c|c|c|c|c|c|c|c|c|}
\hline \multicolumn{2}{|c|}{ O1 } & \multicolumn{2}{c|}{ Rock } & \multirow{2}{*}{ Target } & \multirow{2}{*}{$x l$} & \multirow{2}{*}{ Control } & \multirow{2}{*}{$R$} & \multirow{2}{*}{$R$-range } \\
\hline \hline MME & FIM & MME & FIM & & & & & \\
\hline \hline 1 & 1 & 1 & 1 & 0.65 & 0 & 0.68 & 0.29 & $0.276-0.297$ \\
\hline 1 & 1 & 1 & 1 & 0.65 & 0.12 & 0.68 & 0.29 & $0.283-0.307$ \\
\hline 1 & 1 & 1 & 1 & 0.65 & 0.50 & 0.62 & 0.29 & $0.276-0.296$ \\
\hline 0.06 & 2 & 1 & 1 & 0.6 & 0 & 0.64 & 0.093 & $0.085-0.098$ \\
\hline 0.06 & 2 & 1 & 1 & 0.65 & 0 & 0.69 & 0.107 & $0.097-0.115$ \\
\hline 0.06 & 2 & 1 & 1 & 0.7 & 0 & 0.76 & 0.124 & $0.114-0.123$ \\
\hline 0.06 & 2 & 1 & 1 & 0.65 & 0.50 & 0.65 & 0.107 & $0.093-0.115$ \\
\hline 0.06 & 2 & 1 & 1 & 0.65 & 0.12 & 0.68 & 0.107 & $0.101-0.112$ \\
\hline 0.06 & 2 & 0.812 & 1.2 & 0.65 & 0.12 & 0.68 & 0.104 & $0.093-0.116$ \\
\hline 0.06 & 2 & 0.624 & 1.4 & 0.65 & 0.12 & 0.68 & 0.087 & $0.081-0.094$ \\
\hline 0.06 & 2 & 0.436 & 1.6 & 0.65 & 0.12 & 0.68 & 0.051 & $0.028-0.063$ \\
\hline 0.06 & 2 & 0.248 & 1.8 & 0.65 & 0.12 & 0.68 & $* 1$ & $* 1$ \\
\hline 0.06 & 2 & 0.060 & 2 & 0.65 & 0.12 & 0.68 & $* 2$ & $* 2$ \\
\hline
\end{tabular}

1: The proportion of hybrids at the end of the simulation is $0.45 \%$ (Rock only). $R$-values below roundoff limits. $R$-value at the end of the intervention was 0.080 .

2: Rock hybrids become extinct towards the end of the simulations (almost three years after the end of the intervention). $R$-value at the end of the intervention was 0.016 .

Table 3: Hybridisation levels. O1 mosquitoes present one copy of LG and Rock are hybrids with no deadly gen. Simulations: 156 weeks before treatment, 116 of treatment, 156 weeks after treatment. MME= "Male Mating Efficacy" FIM= "Female Increased Mortality". $x l$ is the proportion of dead larvae, etc. recycled into food (see text), Target (Control) indicates the aimed (actual) proportion of genetically modified eggs, $R$ is the hybridisation degree and $R$-range is given as the extreme values over 10 runs of the simulation.

C. What is the expected ratio between released males and local males necessary to achieve different levels of control?

The ratio ranges from $12: 1$ to $40: 1$ to sustain a target of 0.5 to 0.65 of the eggs having the modified genetics. Ratios larger than $100: 1$ are needed to detect sizeable effects at the beginning of the intervention. This can be compared with the reports in [7] where the test started setting a ratio of $10: 1$ in a comparatively large area but it soon became clear that a smaller (or much smaller) area and a higher ratio $(25: 1)$ were necessary in order to observe some effects. Recall that the simulations assume a closed environment. Field trials are likely to require higher ratios because of the possible immigration of wild adults into the intervention region (see point F.) and the emigration of released mosquitoes.

D. How are the results affected by the limitations of a hypothetical mosquito factory? 
In the simulations we have arbitrarily chosen to limit the production to 100000 modified mosquitoes per week. Some explorations have managed to pass this limit. The present simulations always remain below the limit. In practice, a smaller area requires fewer mosquitoes. Therefore, the production limit is not an issue at this level. Repeatedly hitting the limit would slow down or reduce the possibility of attaining the targets of the treatment.

E. How does recycling of dead larvae and pupae affect the dynamics? Dead individuals could be reprocessed in different ways depending on e.g., temperature, bacterial contents, etc. This issue is not intrinsic to the RIDL-SIT technique apart from the fact that this or any other technique must act on a given natural environment. The model allows for sensing this issue to some extent. The effects appear to be less important regarding the genetic diffusion but sensibly larger regarding the temporary reduction in subpopulation sizes during the treatments.

F. Is it possible to eliminate the mosquito?

The model does not consider immigration (or emigration) to (from) the intervention region. Under these conditions, the targeted treatment will not eliminate the mosquito but rather replace it with some hybrid strain. Simulations releasing weekly all the production of the factory suggest that a 100\% Rockefeller strain with two copies of the LG may replace the wild population (remaining stable) if the treatment is long enough (see Appendix D). Analytical results confirm this observation. Population levels are expected to be somewhat smaller and hence mosquitoes larger than in the untreated situation.

G. How does the technique compare to other infrastructural measures when it comes to costs, permanent benefits, risks and mosquito populations?

The model does not contemplate social and economic costs (in part because of lack of information). The simulations suggest that the technique will become a permanent patch, this is, there will be no end to the release programme and as such it represents a structural cost that should be compared with other long-term policies (such as running water, a sewage system, proper handling of water reservoirs, etc.) that set the possibility of a healthier environment.

\section{Comparison with field observations}

Since the initial submission of the present work, two relevant articles reporting on the evolution of Aedes aegypti populations during and after interventions have been published. It is relevant then to compare quantitatively the field observations with our calculations. 
The first work, [70], follows the release of genetically modified mosquitoes in Jacobina and Juazeiro, two locations in Brazil. The reported reduction in egg population is of $70 \%$, and we will compare their results with our runs for a $65 \%$ target (achieving 68\% reduction). Releases lasted for 10 months (Juazeiro) and 27 months (Jacobina). The authors report that reduction of the population lasted for 17 weeks and 32 weeks after the end of the releases. Actually, the data for Jacobina presents the first important peak 14 weeks after the end of the releases. As the authors discuss, these differences are expected because the times at which the intervention ceased corresponds to different weather conditions for each case, namely a favourable season (rainy, Juazeiro) and an unfavourable one (dry, Jacobina). The present model for the $65 \%$ reduction target estimates 13 18 weeks for a full recovery of the population under optimal weather conditions, the shorter times achieved when $O 1$ adults are considered unfitted (the results present little sensibility to the fitness of Rock hybrid type). In our model, a decrease of $90 \%$ in the number of females carrying one or two LG's is observed after 70 days ( $\sim 2.5$ months) (for $O 1$ unfitted adults but fitted Rock hybrids) while field data reports that "transgenic larvae were detected up to" 2 months (Jacobina) and 5 months (Juazeiro) after the release interruption. The recovery of the population during the rainy (favourable) season observed in the field is somewhat faster than in the model. The eradication of the population with LG is produced by the competition with the wild type, a faster recovery of the latter implies a faster eradication of larvae carrying the LG, thus our overestimation of the time after the intervention when they are detectable is linked to the same causes as the recovery time. When $O 1$ adults are considered fitted, the $O 1$ eggs are no longer present after 154 days, a number harder to consider in agreement with field data.

The second work, [1] , measured hybridisation in Jacobina. We will compare their results with a simulation for a 27 months intervention (116 weeks) at $65 \%$ reduction level (see Table 3). They report "The degree of introgression is not trivial. Depending on sample and criterion used to define unambiguous introgression, from about $10 \%$ to $60 \%$ of all individuals have some OX513A genome". Their procedure is to compute an $h$-value, namely the fraction of modified genes out of a set of 21770 well validated, biallelic, mendelian genes presenting polymorphism. $h$-values larger than 0.02 or 0.04 are taken to be "unambiguous introgressed individuals". The $h$-value may be compared with the average degree of hybridisation $R$ in our simulations.

The $R$ value corresponds to an upper limit since the migration flux favours a lower level of hybridisation measurable in the field and the simulated intervention considers that the weather was favourable to mosquito development all the time, but this is not true for the case studies. Of the different cases of fitness of the hybrid population studied, we can consider that the introgression simulated with $O 1$ mosquitoes being as fit as wild mosquitoes produces an $R$ value which is too high compared to field results. When mosquitoes carrying the LG are considered unfitted, it is required that the Rock mosquitoes (hybrids with no LG) have a better fitness than the $O 1$ in order to match the observations. From our simulations and the reported field studies it is not possible to conclude that 
the Rock hybrids are poor competitors in front of the Wild type.

In summary, the results are consistent with hybrids carrying the LG being substantially unfit but first generations of Rock hybrids not being substantially different, in terms of fitness, to the Wild type. At this point it is worth to consider Figure 2 in [70] where an increase in population is observed during the weeks 18 (2013) and 16 (2014) during the intervention time, and an increase in population after week 16 (2014), when the intervention had already ended. This is, in the field data, the pre-intervention population is lower than the postintervention population. There are a number of hypotheses that can account for such changes, and they are worth to be investigated, such as: Changing weather conditions, improved genetics, changes in standard control measures applied and changes in people's behaviour (relaxing environmental sanity).

Considering the limited information available about the local and genetically modified populations, that the trials were performed in a heterogeneous and open area in contrast with the model, and also that weather data was not introduced in our simulations (and is not available), the performance of our minimal-model must be considered fairly good since it produces values in the same range as the corresponding measurements for all the variables considered.

\section{Final discussion and conclusions}

We have introduced a minimal model that allows study of hybridisation produced by interventions with a particular form of genetically modified mosquitoes. Several dynamically relevant factors had to be omitted because of the lack of empirical information. In this respect, the minimal model can serve as the basis of a more detailed model when, and if, the information becomes available.

Introgression of genes (as an independent action) in a population subject to natural selection results always in a population equally or better adapted than the pre-existing population. This is a basic theorem (supported on the mathematics behind genetic propagation) in as much as fitness can be associated with the genetics of the population. Only the quality and quantity of the improvement remains to be ascertained in each situation. The same theorem indicates that the result of lowering genetic variability will in general decrease fitness, the limiting case being a null decrease. Hence, if controlling the population with a SIT technique results in a decrease of variability, the total outcome of the RIDL-SIT experiment is completely uncertain.

When results from the model are compared to recent interventions we observe that they are quantitatively compatible when hybrids carrying the LG are considered to have a mating efficiency equal to mosquitoes carrying two copies of the LG and an increased mortality rate, while hybrids carrying no LG are closer in mating and survival to the wild population. This situation implies that if $F 1$ males (all of them pertaining to the $O 1$ class of the model) are as unfitted as released males and $F 1$ females (and all members of the $O 1$ class) die twice as faster that wild females (an exaggeration), genetic diffusion still reaches important levels. The fitness relative to the Wild population cannot be 
asserted by the present methods. The intervention is not self-cleaning and will leave behind genetic contamination with the unavoidable consequences stated by the theorem just mentioned.

The present model is a "proof of concept" rather than a "production model". One of the goals of this work has been to show how to model the problem of genetic diffusion and to highlight its relevance. Mosquito models can be highly elaborated and as such they are sensible to local differences in populations or environment. A compartmental model intending to forecast the outcome of an intervention can be conceived by merging the modelling strategies developed in this work into a detailed model. The information needed to asses particular interventions includes the reaction norms for developmental treats as a function of genetics (wild (local) mosquitoes, $F 1$ hybrids, $F 1 b$ hybrids), concentration of food and temperature. The mating efficiency of $F 1$ and $F 1 b$ males is needed as well (along with more standard information such as several years of weather records: at least rain and average daily temperature).

The processes of development of models and of introduction of new technologies present some parallels that are worth discussing. In the first place, both processes respond to the different attitudes towards knowledge and learning, or more generally to the epistemic frame(s) in action.

From pre-modelling (intuition-based) conclusions we enter the realm of theoretical conclusions based on mathematical models. A first stage in model development can be called the chimeric level where the modelled mosquito only roughly resembles the real one (take this expression as self-criticism [68, 23]), but at least the time-scales and life-cycle characteristics are present. Models mature into ecologically oriented models [56, 41, 59] fundamentally including phenotypic plasticity [72] and focusing on one strain at a time (subpopulations of mosquitoes in different cities may be strikingly different [21, 22] and even within the same city [73], different genetics is associated to different breeding sites) and may further progress towards evolutionary models [74] accounting for the evolution of phenotypic plasticity. In this higher level we still have everything to learn.

In parallel, technologies start as laboratory tests, essentially ignorant of the hazards and singularities of life outside the laboratory. At this level, technologies are short-sighted; they cannot look ahead the time and space scales of the controlled laboratory tests. In order to mature into reliable tools, they too have to develop, including insight about the mutual influences between technologies and environment. This triggers the need of a research programme and an action policy which eventually may outcome a strategy to handle the challenges of the environment and of (co)evolution.

The perspectives of the RIDL-SIT technique in Aedes aegypti considered before this work are clearly at the first stage. We have pointed out in the previous sections issues about missing information on both the life-cycle of the modified mosquitoes and on their interaction with the environment and with the wild strain. Moreover, already at the level of bibliographical background research we note that important requirements mentioned e.g., in [4] are not fulfilled. 
The information so far produced corresponds to the development of the technology and extrapolations of laboratory results based upon intuitions. We have termed this epistemic frame the technological frame [75, 76]. This issue has been discussed as: "typological versus population thinking in biology" as well [77]. Typological thinking usually implies the fundamental decision of not exploring the differences between a wild environment and the laboratory.

In this work we implemented an environment-aware model of the life-cycle of a strain of Aedes aegypti compatible with field observations [56, 41, 59] also introducing some genetics since the issue at stake with the RIDL-SIT technique is to take advantage of genetic differences between mosquito strains. We have demonstrated that, with the help of mathematical models, it is possible to detect some of the information needed to make less risky decisions than just "trial and error" (which has been the method of technology in all civilisations but it is not the method of science), and that there is more to monitor than the success of the method considered only in the restricted terms of the developers of the technology. The model suggests that not even in a minimal area protected from immigration of wild specimens the technique is able to eliminate the mosquito population. Even worse, the output of the treatment is to produce a new, hybrid, mosquito strain, whose characteristics are unknown. The RIDL-SIT strategy can be viewed as a technological patch for an unsustainable control programme, a conclusion that has been reached previously from social considerations [14]. Indeed, the results of this work indicate that there is a long path that needs to be travelled before moving into field tests.

The limited nature of the model manifests the need of understanding evolutionary issues in order to proceed further: How might the mosquito change under selective pressure? How will the differences between hybrid and wild mosquitoes manifest themselves? The results of hybridisation cannot be forecast in the present context of lack of information, yet this difficulty should not be rephrased as "there are no consequences expected from hybridisation". In all cases, the larger genetic pool available will result in a better matching of the local forms of the mosquito to the environment. This situation opens a large range of possibilities we only can speculate about. They go from a return to the pre-release equivalent epidemic risk to situations of increased risk. Let us consider two possible mechanisms for the latter. If the larger fertility provided by the Rockefeller background of the released mosquitoes results in larger fertility of the new hybrid population established in place of the original wild population, without altering the available biomass of food, a larger number of adults of smaller size than the original wild population is to be expected. A larger number of vectors facilitates the propagation of mosquito transmitted diseases, while the favourable or unfavourable incidence of the size of the vector is a matter of discussion [78]. In the same form, a co-evolution of bacteria in which Ae ae. feeds prompted by the increase in mortality in the breeding sites is expected to result in substantially larger populations because of the sensitivity detected to "recycling" dead pupae and larvae. Thus, an "arms race" scenario might arise (increased population retaliated by increased releases). The epidemiological situation after the intervention could then be worse than before 
the intervention. Some detailed models like SkeeterBuster predict overshooting of mosquito populations going above the equilibrium value after interventions, others like aedesBA do not [55], it is a matter under discussion that depends on modelling decisions under uncertainty. We must conclude that the possibility of a renewed risk for epidemics after the intervention has ceased should be taken into account. The observed increases in population during and after the Juazeiro tests should be addressed, they cannot be ignored.

This work, as much as previous experience in modelling Aedes aegypti populations, indicates that local differences in environment and mosquito's phenotype are reflected in the population dynamics in sensible forms. This observation warns us about the risks of attributing to the species what is in fact the result of the genetics and environment. Mosquito strains are different, they interact in (so far) unexpected ways and are subject to a large, and mostly unknown today, number of influences. The technological frame (or typological thinking) excels at the time of proposing interventions but ecological or population thinking must be used in evaluating the proposals. In the same direction, it would be a mistake to extrapolate the situation studied in this work to other conceivable cases of RIDL-SIT; the evaluation of every case must be performed based on actual measurements of biological traits, and such experimental values should be produced. Possible progress is then conceived as a dialogue between both biological views. The present work indicates that mathematical modelling can be an important form to explore proposals and possible outcomes.

The standard analogy between controlling fruit flies and controlling mosquitoes such as Ae ae. is not a very close one from an ecological point of view. A very obvious difference is the landscape, going from agricultural to urban, yet a deeper difference consists in that $A e$ ae. is an established domestic insect limited only by the environment, while the fruit fly, when production has not been abandoned, is not limited by the available environment, but rather the fly is just attempting to colonise it, being in short number relative to the carrying capacity. Current knowledge in SIT indicates that "releasing sterile insects routinely in certain areas may be more expedient to prevent establishment of major pests than eliminating them after they become established" [16].

\section{Acknowledgements}

HGS acknowledges support from the University of Buenos Aires under grants 20020130100778BA and 20020130100361BA, as well as support from the MOSTICAW (STIC-AMSUD) collaboration. MAN acknowledges support from SveFUM and from Kungliga Fysiografiska Sällskapet i Lund.

\section{References}

[1] Luke Alphey, Mark Benedict, Romeo Bellini, Gary G Clark, David A Dame, Mike W Service, and Stephen L Dobson. Sterile-insect methods for con- 
trol of mosquito-borne diseases: an analysis. Vector-Borne and Zoonotic Diseases, 10(3):295-311, 2010.

[2] HB Morlan, EM McCray Jr, and JW Kilpatrick. Field tests with sexually sterile males for control of Aedes aegypti. Mosquito News, 22(3):295-300, 1962.

[3] Peng Gong, Matthew J Epton, Guoliang Fu, Sarah Scaife, Alexandra Hiscox, Kirsty C Condon, George C Condon, Neil I Morrison, David W Kelly, Tarig Dafa'alla, et al. A dominant lethal genetic system for autocidal control of the mediterranean fruitfly. Nature biotechnology, 23(4):453, 2005.

[4] Mark Q Benedict and Alan S Robinson. The first releases of transgenic mosquitoes: an argument for the sterile insect technique. Trends in parasitology, 19(8):349-355, 2003.

[5] Hoang Kim Phuc, Morten H Andreasen, Rosemary S Burton, Céline Vass, Matthew J Epton, Gavin Pape, Guoliang Fu, Kirsty C Condon, Sarah Scaife, Christl A Donnelly, et al. Late-acting dominant lethal genetic systems and mosquito control. BMC Biology, 5(1):11, 2007.

[6] Angela F Harris, Derric Nimmo, Andrew R McKemey, Nick Kelly, Sarah Scaife, Christl A Donnelly, Camilla Beech, William D Petrie, and Luke Alphey. Field performance of engineered male mosquitoes. Nature biotechnology, 29(11):1034-1037, 2011.

[7] Angela F Harris, Andrew R McKemey, Derric Nimmo, Zoe Curtis, Isaac Black, Siân A Morgan, Marco Neira Oviedo, Renaud Lacroix, Neil Naish, Neil I Morrison, et al. Successful suppression of a field mosquito population by sustained release of engineered male mosquitoes. Nature biotechnology, 30(9):828-830, 2012.

[8] Renaud Lacroix, Andrew R McKemey, Norzahira Raduan, Lim Kwee Wee, Wong Hong Ming, Teoh Guat Ney, Siti Rahidah AA, Sawaluddin Salman, Selvi Subramaniam, Oreenaiza Nordin, et al. Open field release of genetically engineered sterile male Aedes aegypti in Malaysia. PloS one, 7(8): e42771, 2012.

[9] Danilo O Carvalho, Andrew R McKemey, Luiza Garziera, Renaud Lacroix, Christl A Donnelly, Luke Alphey, Aldo Malavasi, and Margareth L Capurro. Suppression of a field population of Aedes aegypti in Brazil by sustained release of transgenic male mosquitoes. PLoS Negl Trop Dis, 9(7): e0003864, 2015.

[10] Peter Winskill, Danilo O Carvalho, Margareth L Capurro, Luke Alphey, Christl A Donnelly, and Andrew R McKemey. Dispersal of engineered male Aedes aegypti mosquitoes. PLoS neglected tropical diseases, 9(11): e0004156, 2015. 
[11] World Health Organization. Promising new tools to fight Aedes mosquitoes. Bulletin of the World Health Organ 2016, 94:562-563, 2016. doi: http: //dx.doi.org/10.2471/BLT.16.020816.

[12] Center for Veterinary Medicine. Environmental assessment for investigational use of Aedes aegypti ox513a. Technical report, United States Food and Drug Administration, Department of Health and Human Services, 2016.

[13] The Entomological Society of America (ESA) and Sociedade Entomológica do Brasil (SEB). Aedes aegypti and the zika virus: Symptoms of a wider problem. In Summit on the Aedes aegypti Crisis in the Americas, 2016.

[14] Luisa Reis-Castro and Kim Hendrickx. Winged promises: exploring the discourse on transgenic mosquitoes in Brazil. Technology in Society, 35(2): 118-128, 2013.

[15] Walther Enkerlin, José Manuel Gutiérrez-Ruelas, Antonio Villaseñor Cortes, Edgar Cotoc Roldan, David Midgarden, Estuardo Lira, Jose Luis Zavala López, Jorge Hendrichs, Pablo Liedo, and Francisco Javier Trujillo Arriaga. Area freedom in Mexico from Mediterranean fruit fly (diptera: Tephritidae): a review of over 30 years of a successful containment program using an integrated area-wide sit approach. Florida entomologist, 98 (2):665-681, 2015.

[16] J Hendrichs, MJB Vreysen, WR Enkerlin, and JP Cayol. Strategic options in using sterile insects for area-wide integrated pest management. In Sterile Insect Technique, pages 563-600. Springer, 2005.

[17] W Klassen and CF Curtis. History of the sterile insect technique. In Sterile Insect Technique, pages 3-36. Springer, 2005.

[18] DE Weidhaas, SG Breeland, CS Lofgren, DA Dame, and R Kaiser. Release of chemosterilized males for the control of anopheles albimanus in El Salvador. The American journal of tropical medicine and hygiene, 23(2): 298-308, 1974.

[19] David J Rogers and Sarah E Randolph. From a case study to a theoretical basis for tsetse control. International Journal of Tropical Insect Science, 5 (5):419-423, 1984.

[20] Stephen L Dobson, Charles W Fox, and Francis M Jiggins. The effect of wolbachia-induced cytoplasmic incompatibility on host population size in natural and manipulated systems. Proceedings of the Royal Society of London B: Biological Sciences, 269(1490):437-445, 2002.

[21] Edmundo Fabricio Tejerina, Francisco Felipe Ludueña Almeida, and Walter Ricardo Almirón. Bionomics of Aedes aegypti subpopulations (Diptera: 
Culicidae) from Misiones province, northeastern Argentina. Acta Tropica, 109(1):45-49, Jan 2009. doi: 10.1016/j.actatropica.2008.09.014. URL http://dx.doi.org/10.1016/j.actatropica.2008.09.014.

[22] Marta Gladys Grech, Francisco Ludueña-Almeida, and Walter Ricardo Almirón. Bionomics of Aedes aegypti subpopulations (Diptera: Culicidae) from Argentina. Journal of Vector Ecology, 35(2):277-285, Dec 2010. doi: 10.1111/j.1948-7134.2010.00083.x. URL http://dx.doi.org/10.1111/j. $1948-7134.2010 .00083 . x$.

[23] M Otero, N Schweigmann, and H G Solari. A stochastic spatial dynamical model for Aedes aegypti. Bulletin of Mathematical Biology, 70:1297-1325, 2008.

[24] P Bergero, C Ruggerio, R Lombardo, N Schweigmann, and H Solari. Dispersal of Aedes aegypti: field study in temperate areas and statistical approach. Journal of Vector Borne Diseases, 50:163-170, 2013. Journal of Vector Borne Diseases.

[25] P J H Sharpe and D W DeMichele. Reaction kinetics of poikilotherm development. Journal of Theoretical Biology, 64:649-670, 1977.

[26] L. M. Rueda, K. J. Patel, R. C. Axtell, and R. E. Stinner. Temperaturedependent development and survival rates of Culex quinquefasciatus and Aedes aegypti (Diptera: Culicidae). Journal of Medical Entomology, 27: 892-898, 1990.

[27] Andrea Gloria-Soria, Diego Ayala, Ambicadutt Bheecarry, Olger CalderonArguedas, Dave D Chadee, Marina Chiappero, Maureen Coetzee, Khouaildi Bin Elahee, Ildefonso Fernandez-Salas, Hany A Kamal, Basile Kamgang, Emad I. M. Khater, Laura D. Kramer, Vicki Kramer, Alma Lopez-Solis, Joel Lutomiah, Martins Jr, Maria Victoria Micieli, Christophe Paupy, Alongkot Ponlawat, Nil Rahola, Syed Basit Rasheed, Joshua B. Richardson, Amag A. Saleh, Rosa Maria Sanchez-Casas, Gonçalo Seixas, Carla A. Sousa, Walter J. Tabachnick, Adriana Adriana Troyo, and Jeffrey R. Powell. Global genetic diversity of Aedes aegypti. Molecular ecology, 25(21):5377-5395, 2016.

[28] Dave D Chadee and Raymond Martinez. Aedes aegypti (1.) in Latin American and Caribbean region: With growing evidence for vector adaptation to climate change? Acta tropica, 156:137-143, 2016.

[29] Matthew Hamilton. Population genetics. John Wiley \& Sons, 2011.

[30] J D Gillett. Variation in the hatching-response of Aedes eggs (Diptera: Culicidae). Bulletin of the World Health Organization, 46:241-255, 1955.

[31] J D Gillett. The inherited basis of variation in the hatching response of Aedes eggs (Diptera: Culicidae). Bulletin of the World Health Organization, 46:255-265, 1955. 
[32] J D Gillett. Control of hatching in prediapause eggs of Aedes mosquitoes. Nature, 104:1621-1623, 1959.

[33] J D Gillett, E A Roman, and V Phillips. Erratic hatching in aedes eggs: A new interpretation. Proc. R. Soc. Lond. B, 196:223-232, 1977.

[34] Todd P Livdahl and J S Edgerly. Egg hatching inhibition: Field evidence for population regulation in a treehole mosquito. Ecological Entomology, 12:395-399, 1987.

[35] Janice S Edgerly and Michelle A Marvier. To hatch or not to hatch? egg hatch response to larval density and to larval contact in a treehole mosquito. Ecological Entomology, 17:28-32, 1992.

[36] J S Edgerly, M S Willey, and T P Livdhal. The community ecology of Aedes egg hatching: Implications for mosquito invasion. Ecological Entomology, 18:123-128, 1993.

[37] T. R. E. Southwood, G. Murdie, M. Yasuno, R. J. Tonn, and P. M. Reader. Studies on the life budget of Aedes aegypti in Wat Samphaya Bangkok Thailand. Bulletin of the World Health Organization, 46:211-226, 1972.

[38] D A Focks, D C Haile, E Daniels, and G A Moun. Dynamics life table model for Aedes aegypti: Analysis of the literature and model development. Journal of Medical Entomology, 30:1003-1018, 1993.

[39] Arnaldo Maciá. Differences in performance of Aedes aegypti larvae raised at different densities in tires and ovitraps under field conditions in Argentina. Journal of Vector Ecology, 31(2):371-377, Dec 2006.

[40] A Maciá. Effects of larval crowding on development time, survival and weight at metamorphosis in Aedes aegypti (Diptera: Culicidae). Revista Sociedad Entomológica Argentina, 68 (1-2):107-114, 2009.

[41] Victoria Romeo Aznar, María Sol De Majo, Sylvia Fischer, Mario A Natiello, and Hernán G Solari. A model for the development of Aedes (Stegomyia) aegypti (and other insects) as a function of the available food. Journal of Theoretical Biology, 365:311-324, 2015. URL http://dx.doi.org/10. 1016/j.jtbi.2014.10.016.

[42] J Arrivillaga and R Barrera. Food as a limiting factor for Aedes aegypti in water-storage containers. Journal of Vector Ecology, 29:11-20, 2004.

[43] H. Padmanabha, B. Bolker, C. C. Lord, C. Rubio, and L. P. Lounibos. Food availability alters the effects of larval temperature on aedes aegypti growth. Journal of Medical Entomology, 48(5):974-984, Sep 2011.

[44] Lourdes Esteva and Hyun Mo Yang. Mathematical model to assess the control of aedes aegypti mosquitoes by the sterile insect technique. Mathematical biosciences, 198(2):132-147, 2005. 
[45] Laith Yakob, Luke Alphey, and Michael B Bonsall. Aedes aegypti control: the concomitant role of competition, space and transgenic technologies. Journal of applied Ecology, 45(4):1258-1265, 2008.

[46] Jia Li. Simple stage-structured models for wild and transgenic mosquito populations. Journal of Difference Equations and Applications, 15(4):327$347,2009$.

[47] Steven M White, Pejman Rohani, and Steven M Sait. Modelling pulsed releases for sterile insect techniques: fitness costs of sterile and transgenic males and the effects on mosquito dynamics. Journal of Applied Ecology, 47(6):1329-1339, 2010.

[48] S Seirin Lee, RE Baker, EA Gaffney, and SM White. Modelling aedes aegypti mosquito control via transgenic and sterile insect techniques: Endemics and emerging outbreaks. Journal of theoretical biology, 331:78-90, 2013.

[49] S Seirin Lee, Ruth E Baker, Eamonn A Gaffney, and Steven M White. Optimal barrier zones for stopping the invasion of aedes aegypti mosquitoes via transgenic or sterile insect techniques. Theoretical ecology, 6(4):427-442, 2013.

[50] Krisztian Magori, Mathieu Legros, Molly E. Puente, Dana A. Focks, Thomas W. Scott, Alun L. Lloyd, and Fred Gould. Skeeter buster: A stochastic, spatially explicit modeling tool for studying Aedes aegypti population replacement and population suppression strategies. PLoS Neglected Tropical Diseases, 3(9):e508, 09 2009. doi: 10.1371/journal.pntd.0000508. URL http://dx.doi.org/10.1371\%2Fjournal.pntd.0000508.

[51] Mathieu Legros, Chonggang Xu, Kenichi Okamoto, Thomas W Scott, Amy C Morrison, Alun L Lloyd, and Fred Gould. Assessing the feasibility of controlling aedes aegypti with transgenic methods: a model-based evaluation. PloS one, 7(12):e52235, 2012.

[52] Michael A Robert, Mathieu Legros, Luca Facchinelli, Laura Valerio, Janine M Ramsey, Thomas W Scott, Fred Gould, and Alun L Lloyd. Mathematical models as aids for design and development of experiments: the case of transgenic mosquitoes. Journal of medical entomology, 49(6):1177-1188, 2014.

[53] D A Focks, D C Haile, E Daniels, and G A Mount. Dynamic life table model for Aedes aegypti: Simulations results. Journal of Medical Entomology, 30: 1019-1029, 1993.

[54] Alicia M Ellis, Andres J Garcia, Dana A Focks, Amy C Morrison, and Thomas W Scott. Parameterization and sensitivity analysis of a complex simulation model for mosquito population dynamics, dengue transmission, and their control. American Journal of Tropical Medicine and Hygiene, 85 
(2):257-264, Aug 2011. doi: 10.4269/ajtmh.2011.10-0516. URL http:// dx.doi.org/10.4269/ajtmh.2011.10-0516.

[55] Mathieu Legros, Marcelo Otero, Victoria Romeo Aznar, Hernan Solari, Fred Gould, and Alun L Lloyd. Comparison of two detailed models of aedes aegypti population dynamics. Ecosphere, 7(10), 2016.

[56] V Romeo Aznar, M J Otero, M S de Majo, S Fischer, and H G Solari. Modelling the complex hatching and development of Aedes aegypti in temperated climates. Ecological Modelling, 253:44-55, 2013. URL http://dx. doi.org/10.1016/j.ecolmodel.2012.12.004.

[57] R Durrett. Essentials of Stochastic Processes. Springer Verlag, New York, 2001.

[58] Michael E Gilpin and GAH McClelland. Systems analysis of the yellow fever mosquito Aedes aegypti. Fortschritte der Zoologie, 25:355:388, 1979.

[59] Victoria Romeo Aznar. Biología teórica, modelo y experimentos aplicados al entendimiento de la dinámica poblacional del mosquito Aedes aegypti. $\mathrm{PhD}$ thesis, Facultad de Ciencias Exactas y Naturales, Universidad de Buenos Aires, 2015. URL http://digital.bl.fcen.uba.ar/gsdl-282/cgi-bin/ library.cgi?a=d\&c=tesis\&d=Tesis_5982_RomeoAznar.

[60] A Kolmogoroff. Über die analytischen methoden in der wahrscheinlichkeitsrechnung. Mathematische Annalen, 104:415-458, 1931. ISSN 0025-5831. URL http://dx.doi.org/10.1007/BF01457949, 10.1007/BF01457949.

[61] Willy Feller. On the integro-differential equations of purely discontinuous Markoff processes. Transactions of the American Mathematical Society, 48 (3):488-515, 1940. ISSN 00029947. URL http://www.jstor.org/stable/ 1990095 .

[62] S N Ethier and T G Kurtz. Markov Processes. John Wiley and Sons, New York, 1986.

[63] T G Kurtz. Solutions of ordinary differential equations as limits of pure jump Markov processes. J. Appl. Prob., 7:49-58, 1970.

[64] T. G. Kurtz. Strong approximation theorems for density dependent Markov chains. Stochastic Processes and Applications, 6:223, 1978.

[65] H G Solari and M A Natiello. Linear processes in stochastic population dynamics: Theory and application to insect development. The Scientific World Journal - Journal of Probability and Statistics., ID 873624: 1-15, 2014. URL http://downloads.hindawi.com/journals/tswj/aip/ 873624.pdf.

[66] David G Kendall. Stochastic processes and population growth. Journal of the Royal Statistical Society. Series B (Methodological), 11:230-282, 1949. 
[67] David G. Kendall. An artificial realization of a simple "birth-and-death" process. Journal of the Royal Statistical Society. Series B (Methodological), 12:116-119, 1950.

[68] M Otero, H G Solari, and N Schweigmann. A stochastic population dynamic model for Aedes aegypti: Formulation and application to a city with temperate climate. Bulletin of Mathematical Biology, 68:1945-1974, 2006.

[69] M. Trpis. Dry season survival of Aedes aegypti eggs in various breeding sites in the Dar es Salaam area, Tanzania. Bull. W.H.O., 47:433-437, 1972.

[70] Luiza Garziera, Michelle Cristine Pedrosa, Fabricio Almeida de Souza, Maylen Gómez, Márcia Bento Moreira, Jair Fernandes Virginio, Margareth Lara Capurro, and Danilo Oliveira Carvalho. Effect of interruption of over-flooding releases of transgenic mosquitoes over wild population of a edes aegypti: two case studies in brazil. Entomologia Experimentalis et Applicata, 164(3):327-339, 2017.

[71] Benjamin R Evans, Panayiota Kotsakiozi, Andre Luis Costa-da Silva, Rafaella Sayuri Ioshino, Luiza Garziera, Michele C Pedrosa, Aldo Malavasi, Jair F Virginio, Margareth L Capurro, and Jeffrey R Powell. Transgenic aedes aegypti mosquitoes transfer genes into a natural population. Scientific reports, 9(1):1-6, 2019.

[72] Thomas E Reed, Robin S Waples, Daniel E Schindler, Jeffrey J Hard, and Michael T Kinnison. Phenotypic plasticity and population viability: the importance of environmental predictability. Proc Biol Sci, 277(1699):33913400, Nov 2010. doi: 10.1098/rspb.2010.0771. URL http://dx.doi.org/ 10.1098/rspb.2010.0771.

[73] Christophe Paupy, Ngan Chantha, Karine Huber, Nicolas Lecoz, Jean-marc Reynes, Francois Rodhain, and Anna-Bella Failloux. Influence of breeding sites features on genetic differentiation of Aedes aegypti populations analyzed on a local scale in Phnom Penh municipality of Cambodia. The American journal of tropical medicine and hygiene, 71(1):73-81, 2004.

[74] Samuel M Scheiner. Genetics and evolution of phenotypic plasticity. Annual review of ecology and systematics, 24(1):35-68, 1993.

[75] Hernán G Solari. Recognizing the epistemic frame in opportunity of the zika pandemic. In Congrès mondial pour la pensée complexe (Les défis d'un monde globalisé), 2016. URL https://www.reseau-canope. $\mathrm{fr} / \mathrm{fileadmin/user}$ _upload/Projets/pensee_complexe/solari_ recognizing_epistemic_frame_opportunity_zika_pandemic.pdf.

[76] Hernán G Solari. Epistemic considerations on research about flavivirus induced fevers. 2017. URL http://users.df.uba.ar/solari/papers/ sola17-ddenuefever.pdf. 
[77] Jeffrey Powell. Genetic variation in insect vectors: Death of typology? Insects, 9(4):139, 2018.

[78] Steven A Juliano, Gabriel Sylvestre Ribeiro, Rafael Maciel-de Freitas, Márcia G Castro, Claudia Codeço, Ricardo Lourenço-de Oliveira, and L Philip Lounibos. She'sa femme fatale: low-density larval development produces good disease vectors. Memorias do Instituto Oswaldo Cruz, 109(8):10701077, 2014.

[79] PF Verhulst. Notice sur la loi que la population suit dans son accroissement. Correspondence Mathematic et Physic, 10:113-121, 1838.

[80] H G Solari and M A Natiello. Stochastic population dynamics: the Poisson approximation. Physical Review E, 67:031918, 2003. 


\section{A Lethal-gene enhanced mortality at pupae level}

The assumptions behind the modified death and emergence rates for pupae are as follows. For the model, pupae can undergo only two events, namely emergence $m_{p \rightarrow a}$ and death $m_{p}$. We assume that the time to the next event is not altered with respect to the wild population, so in both cases this time responds to an exponential distribution with parameter $R_{\text {pupa }}=m_{p \rightarrow a}+m_{p}=m_{p l \rightarrow a}+m_{p l}$ (where $l$ stands for "lethal"). Further, we assume that $5 \%$ of pupae carrying the LG emerge while the other $95 \%$ dies. Hence,

$$
\frac{m_{p l \rightarrow a}}{m_{p l \rightarrow a}+m_{p l}}=0.05
$$

and therefore

$$
\frac{m_{p l \rightarrow a}+m_{p l}}{m_{p l \rightarrow a}}=20 \quad \text { or } \quad m_{p l}=19 m_{p l \rightarrow a}
$$

Hence,

$$
\begin{aligned}
m_{p l \rightarrow a} & =\frac{1}{20}\left(m_{p \rightarrow a}+m_{p}\right), \quad \text { and } \\
m_{p l} & =\frac{19}{20}\left(m_{p \rightarrow a}+m_{p}\right) .
\end{aligned}
$$

\section{B Deterministic equilibrium population}

Deterministic population equations as those are frequently seen in epidemiological modelling, have an older history [79] than the stochastic approach. However, deterministic models have a more limited value, since they average away relevant issues of the dynamics. The most conspicuous limitation is that these models cannot properly describe few individuals, as would be the case in problems where there is a potential extinction risk (such as ours). In certain situations [62, 80] the deterministic dynamics can be seen as a sort of largepopulation limit of the stochastic approach. However, since the wild population is not spontaneously facing extinction, the deterministic equilibrium values are a reasonable starting point for our simulations.

To produce deterministic equations out of the stochastic setup amounts to combine the rates $W_{\alpha}$ and associated modifications $\delta_{j}^{\alpha}$ populationwise.

The general expression for the deterministic dynamics of each population species in the absence of released individuals reads,

$$
\frac{d X_{j}}{d t}=\sum_{\alpha} W_{\alpha}\left(X_{1}, \cdots, X_{n}\right) \delta_{j}^{\alpha}=\sum_{\alpha} m_{\alpha}^{i} X_{i} \delta_{j}^{\alpha}
$$

where the sum over $\alpha$ is relevant only for those events modifying population $X_{j}$ and $X_{i}$ is the specific subpopulation associated to the event with coefficient $m_{\alpha}^{i}$. At this point recall that fecundation is not treated as an event, i.e., it is assumed to occur in a time-scale that is negligible in front of the life-span of 
adult females. Letting $E, L, P, F$ denote the size of the wild populations of eggs, larvae, pupae and females, we obtain,

$$
\begin{aligned}
\frac{d E}{d t} & =m_{\text {ovi }} c_{\text {lay }} F T\left(\frac{P_{f}}{C_{f}}\right) F-\left(m_{e \rightarrow l}+m_{e}\right) E \\
\frac{d L}{d t} & =m_{e \rightarrow l} E-L E\left(\frac{P_{f}}{C_{f}}\right) L \\
\frac{d P}{d t} & =L E\left(\frac{P_{f}}{C_{f}}\right)\left(1-M L\left(\frac{P_{f}}{C_{f}}\right)\right) L-0.5787 P \\
\frac{d F}{d t} & =\frac{1}{2} 0.5787\left(1-M L\left(\frac{P_{f}}{C_{f}}\right)\right) P-\frac{0.04}{1-M L\left(\frac{P_{f}}{C_{f}}\right)} F
\end{aligned}
$$

The food equilibrium condition influences all equations and also the larvae population $L$. Being the deterministic system quasilinear [65] in the populations, the equilibrium is obtained when the determinant of the coefficient matrix is zero, determining in such a form the food level of the equilibrium. In terms of exponential races, the equilibrium is independent of the average time required for the race and depends only on the probability of occurrence of one or another outcome. The population number is determined by the production of food by the environment. The equilibrium values were obtained solving the equilibrium food dynamics to obtain the equilibrium $\frac{P_{f}}{C_{f}}$ value and the equilibrium larvae population $L$. Subsequently, all other populations were obtained by solving $\frac{d E}{d t}=0, \frac{d P}{d t}=0, \frac{d F}{d t}=0$ as a function of $L$ and $\frac{P_{f}}{C_{f}}$.

\section{Reaction norms determined for this work}

The data available from the experiment reported in [41, 59] was used to produce the estimates for the dependence of body size (surrogated by wing length) and the rate for events (pupation or death) as larvae. The results are displayed in Figure 5. The data displays clearly two different responses to food depletion: the starvation region where the probability of mortality increases but the average time for the next event in the larvae compartment does not change significantly; and the scarcity region where mortality is not increased but the average time between events (the reciprocal of the rate) changes monotonically. The left panel of Figure 5 shows the wing length as a function of the logarithm of the fraction of optimal food density. Wing length, body size and fertility are monotonically related. The right panel shows the event rate for larvae as a function of the same environmental variable.

\section{Constant vs targeted release}

The goal of control strategies is to reduce mosquito populations to a level in which epidemic outbreaks are not possible. Releasing genetically modified mos- 

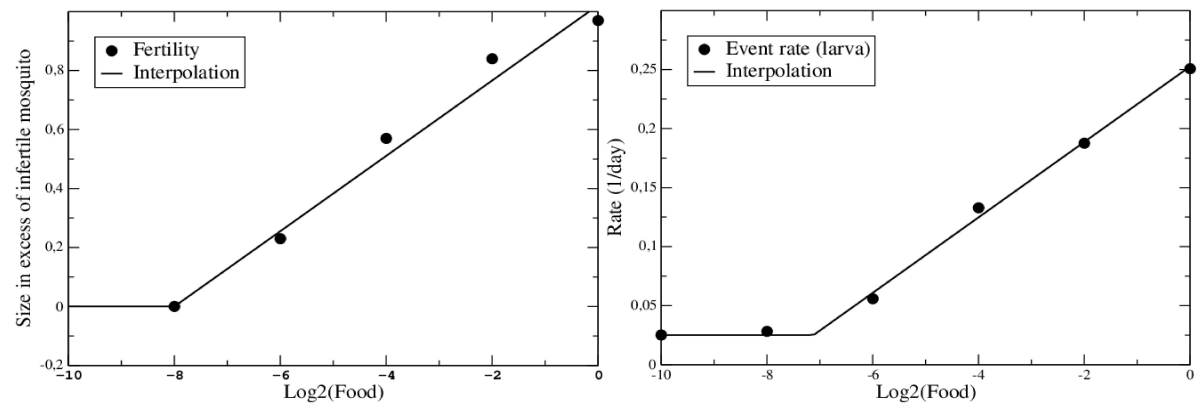

Figure 5: Reaction norms for fertility and event rate (death plus pupation) for larvae. (No females emerged for the $2^{-10}$ treatment).

quitoes in excess of those needed to reach the target represents a misuse of costly resources. A compromise must be taken between the area under control and the degree of control (meaning the number of mosquitoes per human being for example).

In contrast, in a trial phase, one may want to test how to achieve maximal effect. To depict this situation we simulated five "large" constant weekly releases (larger or much larger than the maintenance release targeted in Figures 2,3 and (4), acting under two years, for the case of $12 \%$ food recycling. Only for the larger values the wild and hybrid (with no LG) populations are outnumbered to extinction in the present modelling conditions (closed environment with no immigration/emigration of individuals, no heterogeneities), while a smaller population of $100 \%$ Rockefeller with 2 LG individuals persists for over three years after the intervention. Results are displayed in Figure 6. However, this situation is unrealistic in field conditions where immigration/emigration cannot be controlled. 

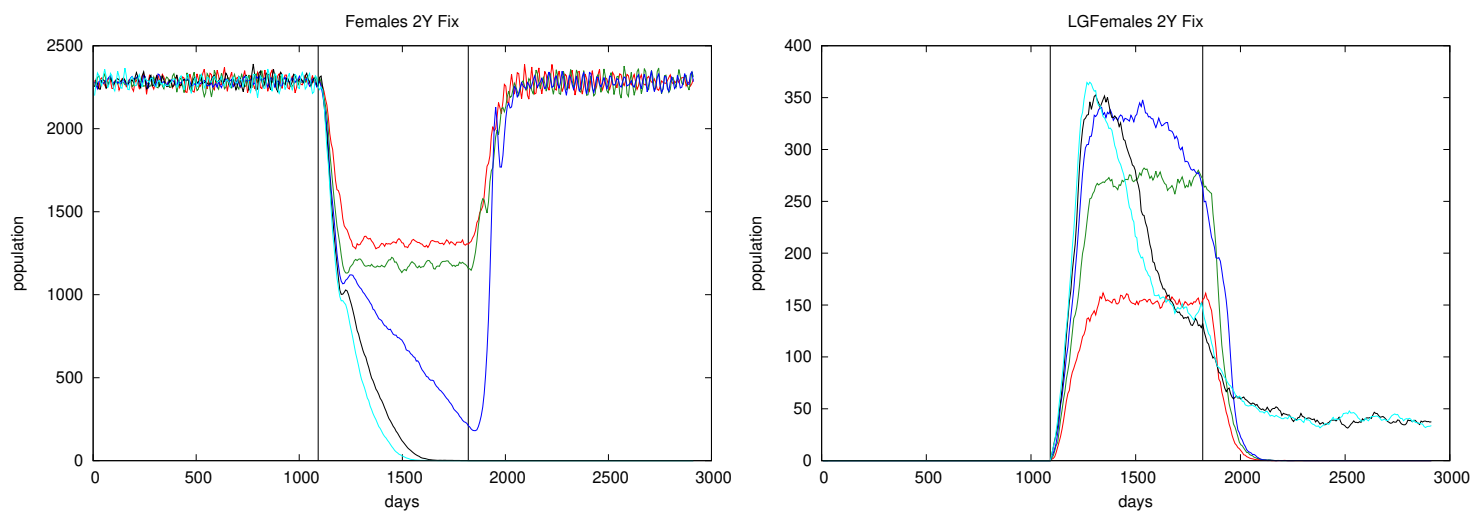

Figure 6: Females with no LG (left) and with 2 LG (right) for constant weekly release of 10000 (red) to 50000 (light blue) individuals (green: 20000, dark blue 30000, black 40000 individuals). 